\title{
Russian Federation: Statistical Appendix
}

This Statistical Appendix paper for the Russian Federation was prepared by a staff team of the International Monetary Fund as background documentation for the periodic consultation with the member country. It is based on the information available at the time it was completed on August 5, 2004. The views expressed in this document are those of the staff team and do not necessarily reflect the views of the government of the Russian Federation or the Executive Board of the IMF.

The policy of publication of staff reports and other documents by the IMF allows for the deletion of market-sensitive information.

To assist the IMF in evaluating the publication policy, reader comments are invited and may be sent by e-mail to publicationpolicy@imf.org.

Copies of this report are available to the public from

International Monetary Fund • Publication Services

$70019^{\text {th }}$ Street, N.W. • Washington, D.C. 20431

Telephone: (202) 623-7430 • Telefax: (202) 623-7201

E-mail: publications@imf.org Internet: http://www.imf.org

Price: $\$ 15.00$ a copy

\section{International Monetary Fund Washington, D.C.}





\title{
INTERNATIONAL MONETARY FUND
}

\author{
RUSSIAN FEDERATION \\ Statistical Appendix \\ Prepared by Aidar Abdychev, Franziska Ohnsorge, Nienke Oomes (all EUR), \\ Antonio Spilimbergo (FAD), Subir Lall (PDR) \\ Approved by European Department
}

August 5, 2004

Contents

Page

Basic Data 3

Statistical Appendix Tables

1. Selected Indicators of Economic Activity, 1997-2003 ….................................... 4

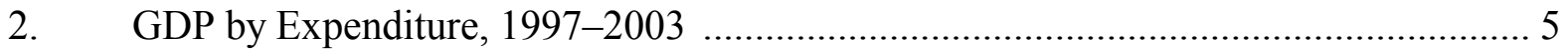

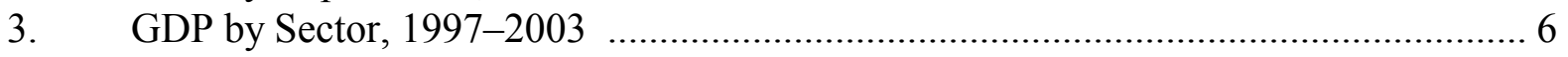

4. Gross Industrial Output by Sector, 1997-2003 .................................................... 7

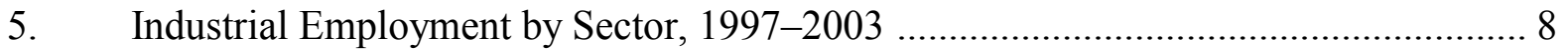

6. Employment by Sector, 1997-2003 …................................................................ 9

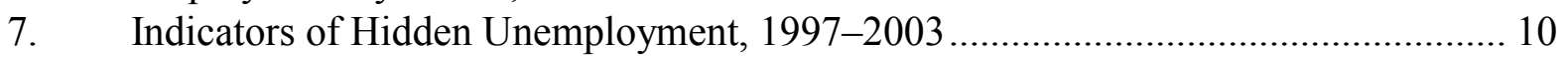

8. Selected Labor Market Indicators, 1997-2003 ................................................. 11

9. Unemployment Rate by Regions (ILO methodology), 1997-2003 …...................... 12

10. Migration Between the Regions of Russia, 1997-2003 ....................................... 14

11. Consumer Price Inflation and CPI Weights, 1997-2003 ....................................... 16

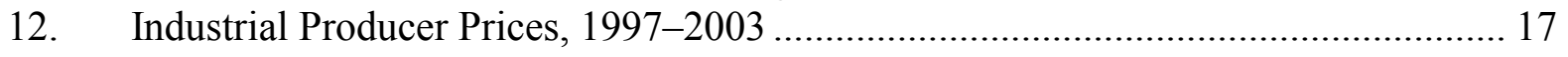

13. Wages, Pensions, and Per Capita Incomes, 1997-2003 ....................................... 18

14. Summary Operations of the Enlarged Government, 1997-2003 ............................ 19

15. Federal Government Budget Execution, 1997-2003 …...................................... 20

16. Regional and Local Government Operations, 1997-2003 .................................... 21

17. Extrabudgetary Fund Operations, 1997-2003 …................................................ 22

18. Monetary Authorities' Accounts, 1998-2003 ...................................................... 23

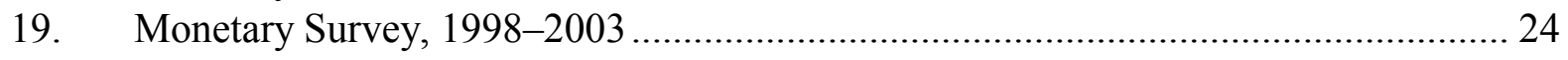

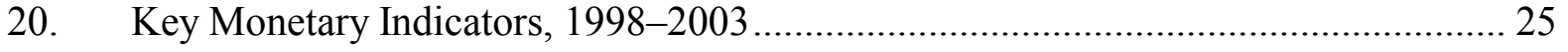

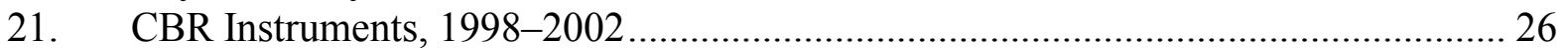

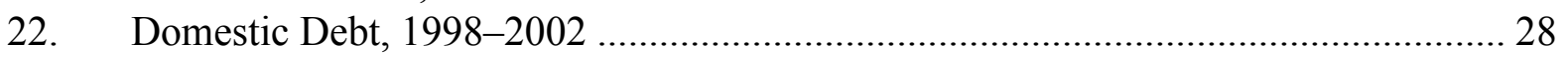

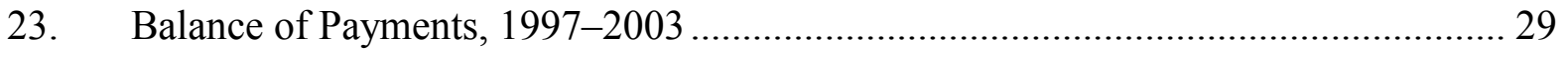

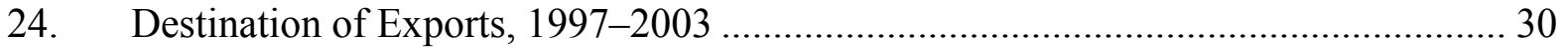


25. Composition of Merchandise Exports, 1997-2003 …......................................... 31

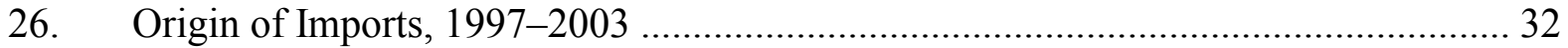

27. Composition of Merchandise Imports, 1997-2003 ............................................... 33

28. Foreign Currency Disbursements to the Federal Government, 1999-2003 .............. 34

29. Nonsovereign/Sector Financial Account, 1997-2003 ....................................... 35

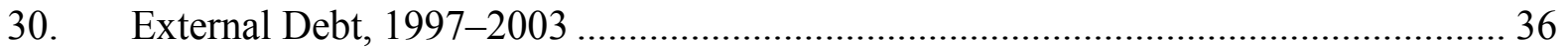




\section{Russian Federation: Basic Data, 1997-2003}

Social and demographic indicators $1 /$

Area

Population (in millions) 2/

Urban (As a percent of total population)

Rate of population growth (Percent per annum)

Life expectancy at birth (Years)

Infant mortality rate (Per 1,000 live births)

Literacy (Percent of population)

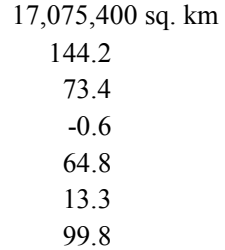

$-0.6$

64.8

13.3

99.8

\begin{tabular}{|c|c|c|c|c|c|c|c|}
\hline & 1997 & 1998 & 1999 & 2000 & 2001 & 2002 & 2003 \\
\hline \multicolumn{8}{|l|}{ Share of gross domestic product (in percent) 3/ } \\
\hline Agriculture 4/ & 6.7 & 5.9 & 7.6 & 6.7 & 6.9 & 6.0 & 5.5 \\
\hline Industry & 29.8 & 30.0 & 31.1 & 31.4 & 28.2 & 27.0 & 27.1 \\
\hline Services & 54.5 & 56.0 & 54.7 & 54.8 & 56.8 & 59.2 & 59.5 \\
\hline \multicolumn{8}{|l|}{ GDP } \\
\hline Nominal GDP (in billions of rubles) & 2,343 & 2,630 & 4,823 & 7,306 & 8,944 & 10,834 & 13,285 \\
\hline Real GDP (percent change) & 1.4 & -5.3 & 6.4 & 10.0 & 5.1 & 4.7 & 7.3 \\
\hline Consumer prices (percent change, annual average) & 14.8 & 27.7 & 85.7 & 20.8 & 21.5 & 15.8 & 13.7 \\
\hline \multicolumn{8}{|l|}{ Enlarged government finances (in billions of rubles) 5/ } \\
\hline Total revenue & 920 & 903 & 1,619 & 2,695 & 3,342 & 4,077 & 4,861 \\
\hline (in percent of GDP) & 39.3 & 34.3 & 33.6 & 36.9 & 37.3 & 37.6 & 36.6 \\
\hline Total expenditure & 1,119 & 1,118 & 1,769 & 2,466 & 3,100 & 4,017 & 4,720 \\
\hline (in percent of GDP) & 47.8 & 42.5 & 36.7 & 33.7 & 34.6 & 37.0 & 35.5 \\
\hline Overall balance & -199 & -215 & -150 & 229 & 243 & 61 & 141 \\
\hline (in percent of GDP) & -8.5 & -8.2 & -3.1 & 3.1 & 2.7 & 0.6 & 1.1 \\
\hline \multicolumn{8}{|l|}{ Money and credit (end of year) } \\
\hline Ruble broad money (in billions of rubles) & 370 & 448 & 705 & 1,144 & 1,603 & 2,120 & 3,213 \\
\hline Velocity of ruble broad money (level) & 7.1 & 7.3 & 8.1 & 6.8 & 6.1 & 5.7 & 4.6 \\
\hline \multicolumn{8}{|l|}{ Balance of payments } \\
\hline Total exports (in billion of US dollars) & 86.9 & 74.4 & 75.6 & 105.0 & 101.9 & 107.2 & 135.9 \\
\hline Total imports (in billion of US dollars) & 72.0 & 58.0 & 39.5 & 44.9 & 53.8 & 61.0 & 75.4 \\
\hline Current account balance (in billion of US dollars) & -2.6 & -2.1 & 22.2 & 44.6 & 33.4 & 30.9 & 35.9 \\
\hline Official reserves (in months of imports of goods and services) & 2.9 & 2.5 & 2.4 & 4.6 & 5.0 & 5.6 & 7.1 \\
\hline Exchange rate (rubles per U.S. dollar, end of year) & 6.0 & 20.7 & 27.0 & 28.2 & 30.1 & 31.8 & 29.5 \\
\hline
\end{tabular}

Sources: Russian authorities; and Fund staff estimates.

1/ Latest available data.

2/ Resident population at January 1, 2004.

3/ GDP share of gross value added by economic sectors, in basic prices, not adjusted for imputed financial services.

4/ Including companies servicing agriculture and forestry.

5/ On commitment basis. 
Table 1. Russian Federation: Selected Indicators of Economic Activity, 1997-2003 1/ (Annual percent change)

\begin{tabular}{lrrrrrrr}
\hline & 1997 & 1998 & 1999 & 2000 & 2001 & 2002 & 2003 \\
\hline Gross domestic product & 1.4 & -5.3 & 6.4 & 10.0 & 5.1 & 4.7 & 7.3 \\
Industrial output & 2.0 & -5.2 & 11.0 & 11.9 & 4.9 & 3.7 & 7.0 \\
Agricultural output & 1.5 & -13.2 & 4.1 & 7.7 & 7.5 & 1.5 & 1.5 \\
Freight transportation 2/ & -3.4 & -3.3 & 5.3 & 5.0 & 3.2 & 5.8 & 7.9 \\
Retail sales & -5.8 & -6.4 & 4.2 & 11.5 & 9.8 & 7.4 & 7.9 \\
Construction & -5.4 & -6.3 & 6.0 & 17.4 & 9.9 & 2.7 & 14.4 \\
\hline
\end{tabular}

Source: Goskomstat.

1/ All figures include estimates of informal (unrecorded) activity.

2/ Turnover of transport companies (including pipelines). 
Table 2. Russian Federation: GDP by Expenditure, 1997-2003

\begin{tabular}{|c|c|c|c|c|c|c|c|}
\hline & 1997 & 1998 & 1999 & 2000 & 2001 & 2002 & 2003 \\
\hline & \multicolumn{7}{|c|}{ (Annual percent change, at constant prices $1 /$ ) } \\
\hline Gross domestic product & 1.4 & -5.3 & 6.4 & 10.0 & 5.1 & 4.7 & 7.3 \\
\hline Consumption & 2.8 & -2.1 & -1.2 & 5.6 & 6.8 & 7.3 & 6.6 \\
\hline Households & 5.0 & -3.4 & -2.9 & 7.3 & 9.5 & 8.9 & 7.9 \\
\hline General government & -2.4 & 1.0 & 3.1 & 2.0 & -0.8 & 2.6 & 2.2 \\
\hline Non-profit institutions & -1.8 & 0.5 & -1.4 & 1.6 & 1.9 & 0.8 & 2.1 \\
\hline Gross investment & -4.1 & -45.2 & -6.6 & 75.2 & 16.7 & -2.2 & 13.5 \\
\hline Capital formation & -7.9 & -12.4 & 6.4 & 18.1 & 10.2 & 3.0 & 12.9 \\
\hline Net exports of goods and services & -5.7 & 131.5 & 79.3 & -15.9 & -13.2 & 1.5 & 3.0 \\
\hline Exports & -0.5 & 1.9 & 11.3 & 9.5 & 4.2 & 9.6 & 13.7 \\
\hline \multirow[t]{2}{*}{ Imports } & 0.4 & -17.4 & -17.1 & 32.4 & 18.7 & 14.6 & 19.5 \\
\hline & \multicolumn{7}{|c|}{ (In percent of GDP) } \\
\hline Consumption & 75.8 & 76.2 & 68.1 & 61.3 & 65.8 & 68.9 & 67.5 \\
\hline Households & 52.7 & 55.6 & 52.4 & 45.1 & 48.3 & 50.0 & 49.4 \\
\hline General government & 21.1 & 18.7 & 14.6 & 15.1 & 16.4 & 17.7 & 16.9 \\
\hline Non-profit institutions & 2.0 & 1.9 & 1.2 & 1.1 & 1.1 & 1.2 & 1.2 \\
\hline Gross Investment & 22.0 & 15.0 & 14.8 & 18.7 & 21.9 & 20.2 & 20.6 \\
\hline Capital formation & 18.3 & 16.1 & 14.4 & 16.9 & 18.9 & 17.9 & 18.2 \\
\hline Changes in inventory & 3.7 & -1.2 & 0.4 & 1.8 & 3.1 & 2.2 & 2.4 \\
\hline Net exports of goods and services & 2.2 & 6.7 & 19.1 & 20.0 & 12.7 & 10.6 & 11.4 \\
\hline Exports & 24.7 & 31.2 & 43.2 & 44.1 & 36.9 & 35.0 & 34.2 \\
\hline Imports & 22.5 & 24.5 & 26.2 & 24.0 & 24.2 & 24.4 & 23.6 \\
\hline
\end{tabular}

Source: Goskomstat, and Fund staff estimates.

1/ Data through 2000 are in constant 1995 prices, data after 2000 are in constant 2000 prices. 
Table 3. Russian Federation: GDP by Sector, 1997-2003 1/

(in percent of GDP)

\begin{tabular}{|c|c|c|c|c|c|c|c|}
\hline & 1997 & 1998 & 1999 & 2000 & 2001 & 2002 & 2003 \\
\hline Agriculture 2/ & 6.7 & 5.9 & 7.6 & 6.7 & 6.9 & 6.0 & 5.5 \\
\hline Industry & 29.8 & 30.0 & 31.1 & 31.4 & 28.2 & 27.0 & 27.1 \\
\hline Construction & 8.4 & 7.4 & 6.1 & 6.6 & 7.4 & 7.0 & 7.1 \\
\hline $\begin{array}{l}\text { Wholesale, retail, foreign trade, public } \\
\text { catering, procurement }\end{array}$ & 18.8 & 20.6 & 23.3 & 23.7 & 22.3 & 22.3 & 22.2 \\
\hline Transportation and communications $3 /$ & 12.5 & 11.0 & 9.6 & 9.1 & 9.1 & 9.3 & 9.4 \\
\hline $\begin{array}{l}\text { Finance, credit, insurance, real estate } \\
\text { operations, science and research, housing, } \\
\text { geology, subsoil resources, exploration, } \\
\text { meteorology, computer services, others }\end{array}$ & 9.2 & 10.1 & 11.1 & 11.7 & 14.0 & 14.7 & 14.9 \\
\hline State administration and defense & 4.8 & 5.3 & 4.4 & 4.2 & 4.7 & 4.9 & 5.0 \\
\hline $\begin{array}{l}\text { Education, culture and art, health care, } \\
\text { physical education \& social security, utilities, } \\
\text { non-production activities services to } \\
\text { households, people's associations }\end{array}$ & 9.8 & 9.7 & 6.8 & 6.6 & 7.4 & 8.8 & 8.8 \\
\hline
\end{tabular}

Source: Goskomstat.

1/ Share of gross value added by economic sector, in basic prices (excludes taxes, includes subsidies; not adjusted for imputed financial services.)

2 / Including companies servicing agriculture and forestry.

3 / Including road infrastructure. 
Table 4. Russian Federation: Gross Industrial Output by Sector, 1997-2003

\begin{tabular}{|c|c|c|c|c|c|c|c|}
\hline & 1997 & 1998 & 1999 & 2000 & 2001 & 2002 & 2003 \\
\hline & \multicolumn{7}{|c|}{ (Percent change, annual average) } \\
\hline Total Industry & 2.0 & -5.2 & 11.0 & 11.9 & 4.9 & 3.7 & 7.0 \\
\hline Electric power generation & -1.8 & -2.3 & -1.2 & 2.3 & 1.6 & -0.7 & 1.0 \\
\hline Fuel & -0.4 & -2.6 & 2.5 & 4.9 & 6.1 & 7.0 & 9.3 \\
\hline Ferrous metallurgy & 0.9 & -7.6 & 16.8 & 15.7 & -0.2 & 3.0 & 8.9 \\
\hline Nonferrous metallurgy & 6.0 & -4.3 & 10.1 & 15.2 & 4.9 & 6.0 & 6.2 \\
\hline Chemicals and petrochemicals $1 /$ & 2.0 & -6.0 & 24.1 & 13.1 & 6.5 & 1.6 & 4.4 \\
\hline Machinery & 3.5 & -8.7 & 17.2 & 20.0 & 7.2 & 2.0 & 9.4 \\
\hline Forestry, timber processing, paper and pulp & -0.4 & 0.4 & 17.8 & 13.4 & 2.6 & 2.4 & 1.5 \\
\hline Construction materials & -4.1 & -6.3 & 10.2 & 13.1 & 5.5 & 3.0 & 6.4 \\
\hline Light industry & -3.9 & -10.3 & 12.3 & 20.9 & 5.0 & -3.4 & -2.3 \\
\hline \multirow[t]{2}{*}{ Food processing } & -2.8 & 0.8 & 3.6 & 14.4 & 8.4 & 6.5 & 5.1 \\
\hline & \multicolumn{7}{|c|}{ (In percent of 1991 level) } \\
\hline Total Industry & 52.5 & 49.8 & 55.2 & 61.8 & 64.8 & 67.2 & 71.9 \\
\hline Electric power generation & 76.6 & 74.8 & 73.9 & 75.6 & 76.9 & 76.3 & 77.1 \\
\hline Fuel & 70.7 & 68.8 & 70.6 & 74.0 & 78.5 & 84.0 & 91.9 \\
\hline Ferrous metallurgy & 60.7 & 56.1 & 65.5 & 75.8 & 75.6 & 77.9 & 84.9 \\
\hline Nonferrous metallurgy & 61.3 & 58.7 & 64.6 & 74.4 & 78.1 & 82.8 & 87.9 \\
\hline Chemicals and petrochemicals & 46.3 & 43.5 & 54.0 & 61.1 & 65.0 & 66.1 & 68.9 \\
\hline Machinery & 40.0 & 36.5 & 42.8 & 51.4 & 55.1 & 56.2 & 61.5 \\
\hline Forestry, timber processing, paper and pulp & 36.9 & 37.1 & 43.7 & 49.5 & 50.8 & 52.1 & 52.8 \\
\hline Construction materials & 32.0 & 29.9 & 33.0 & 37.3 & 39.4 & 40.5 & 43.1 \\
\hline Light industry & 14.0 & 12.6 & 14.1 & 17.1 & 17.9 & 17.3 & 16.9 \\
\hline Food processing & 50.8 & 51.2 & 53.0 & 60.7 & 65.8 & 70.1 & 73.6 \\
\hline
\end{tabular}

Source: Goskomstat.

1/ Excluding the chemical-pharmaceutical industry. 
Table 5. Russian Federation: Employment by Sector, 1997-2003

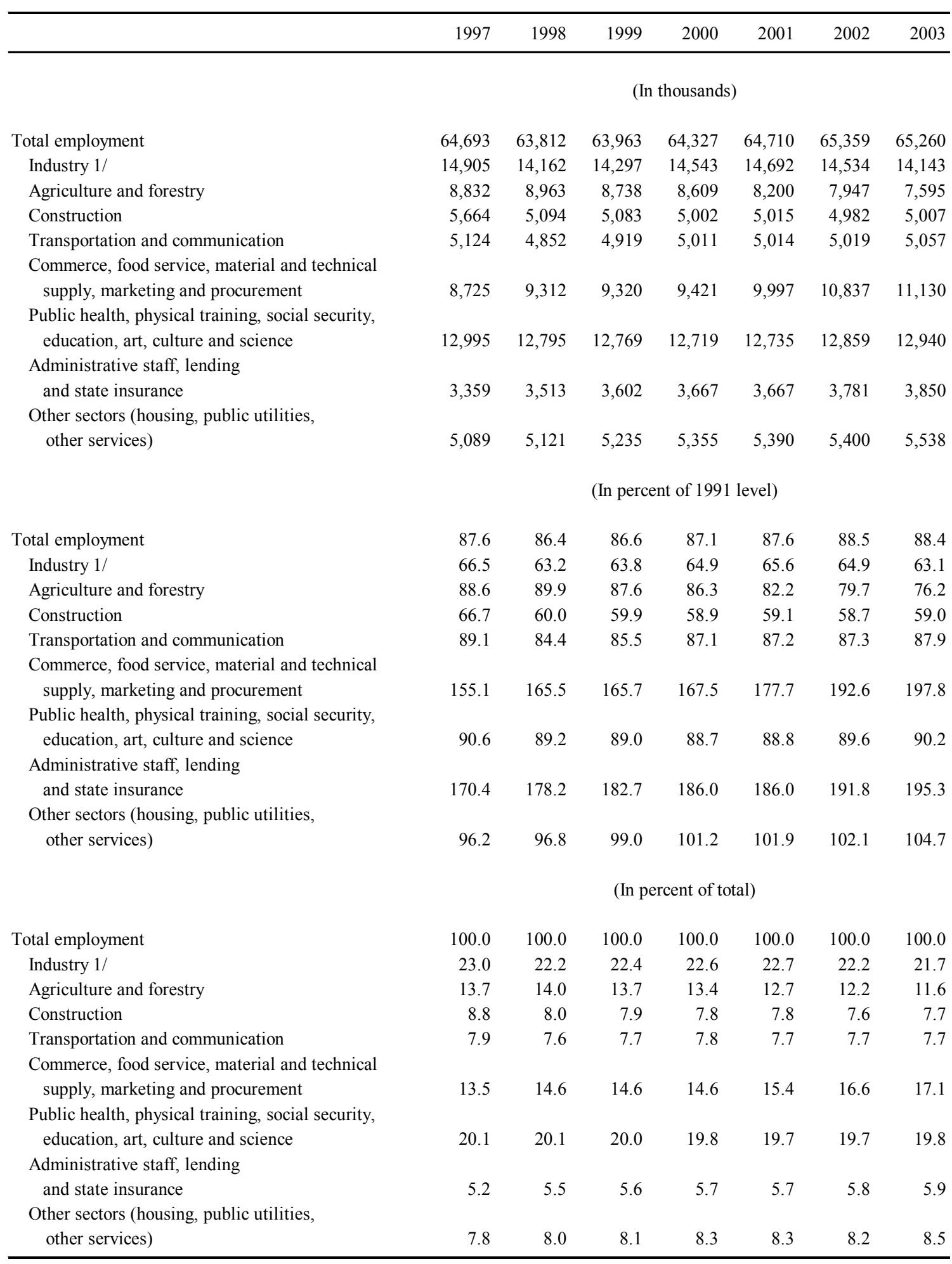

Source: Goskomstat.

1/ Goskomstat estimates, which do not necessarily match the wage fund data in Table 6 . 
Table 6. Russian Federation: Industrial Employment by Sector, 1997-2003 1/

\begin{tabular}{|c|c|c|c|c|c|c|c|}
\hline & 1997 & 1998 & 1999 & 2000 & 2001 & 2002 & 2003 \\
\hline & \multicolumn{7}{|c|}{ (In thousands) } \\
\hline Total industrial employment & 14,009 & 13,173 & 13,077 & 13,294 & 13,282 & 12,886 & 12,296 \\
\hline Electric power generation & 810 & 842 & 880 & 913 & 942 & 928 & 901 \\
\hline Fuel & 821 & 794 & 738 & 730 & 806 & 774 & 818 \\
\hline Ferrous metallurguy & 683 & 673 & 676 & 711 & 727 & 695 & 700 \\
\hline Nonferrous metallurgy & 508 & 480 & 503 & 560 & 582 & 570 & 610 \\
\hline Chemicals and petrochemicals & 821 & 775 & 761 & 785 & 795 & 789 & 769 \\
\hline Machinery & 5,231 & 4,833 & 4,688 & 4,709 & 4,642 & 4,468 & 4,247 \\
\hline Forestry, timber processing, paper and pulp & 1,138 & 1,034 & 1,057 & 1,102 & 1,054 & 1,010 & 886 \\
\hline Construction materials & 783 & 713 & 718 & 684 & 677 & 667 & 576 \\
\hline Light industry & 1,006 & 888 & 863 & 849 & 814 & 765 & 657 \\
\hline Food processing & 1,454 & 1,396 & 1,439 & 1,484 & 1,492 & 1,495 & 1,460 \\
\hline \multirow[t]{2}{*}{ Others } & 754 & 745 & 754 & 767 & 751 & 725 & 672 \\
\hline & \multicolumn{7}{|c|}{ (In percent of 1991 level) } \\
\hline Total industrial employment & 52.5 & 49.8 & 55.2 & 61.8 & 64.8 & 67.2 & 71.9 \\
\hline Electric power generation & 76.6 & 74.8 & 73.9 & 75.6 & 76.9 & 76.3 & 77.1 \\
\hline Fuel & 70.7 & 68.8 & 70.6 & 74.0 & 78.5 & 84.0 & 91.9 \\
\hline Ferrous metallurguy & 60.7 & 56.1 & 65.5 & 75.8 & 75.6 & 77.9 & 84.9 \\
\hline Nonferrous metallurgy & 61.3 & 58.7 & 64.6 & 74.4 & 78.1 & 82.8 & 87.9 \\
\hline Chemicals and petrochemicals & 46.3 & 43.5 & 54.0 & 61.1 & 65.0 & 66.1 & 68.9 \\
\hline Machinery & 40.0 & 36.5 & 42.8 & 51.4 & 55.1 & 56.2 & 61.5 \\
\hline Forestry, timber processing, paper and pulp & 36.9 & 37.1 & 43.7 & 49.5 & 50.8 & 52.1 & 52.8 \\
\hline Construction materials & 32.0 & 29.9 & 33.0 & 37.3 & 39.4 & 40.5 & 43.1 \\
\hline Light industry & 14.0 & 12.6 & 14.1 & 17.1 & 17.9 & 17.3 & 16.9 \\
\hline \multirow[t]{2}{*}{ Food processing } & 50.8 & 51.2 & 53.0 & 60.7 & 65.8 & 70.1 & 73.6 \\
\hline & \multicolumn{7}{|c|}{ (In percent of total) } \\
\hline Total industrial employment & 100.0 & 100.0 & 100.0 & 100.0 & 100.0 & 100.0 & 100.0 \\
\hline Electric power generation & 5.8 & 6.4 & 6.7 & 6.9 & 7.1 & 7.2 & 7.3 \\
\hline Fuel & 5.9 & 6.0 & 5.6 & 5.5 & 6.1 & 6.0 & 6.7 \\
\hline Ferrous metallurguy & 4.9 & 5.1 & 5.2 & 5.3 & 5.5 & 5.4 & 5.7 \\
\hline Nonferrous metallurgy & 3.6 & 3.6 & 3.8 & 4.2 & 4.4 & 4.4 & 5.0 \\
\hline Chemicals and petrochemicals & 5.9 & 5.9 & 5.8 & 5.9 & 6.0 & 6.1 & 6.3 \\
\hline Machinery & 37.3 & 36.7 & 35.8 & 35.4 & 34.9 & 34.7 & 34.5 \\
\hline Forestry, timber processing, paper and pulp & 8.1 & 7.8 & 8.1 & 8.3 & 7.9 & 7.8 & 7.2 \\
\hline Construction materials & 5.6 & 5.4 & 5.5 & 5.1 & 5.1 & 5.2 & 4.7 \\
\hline Light industry & 7.2 & 6.7 & 6.6 & 6.4 & 6.1 & 5.9 & 5.3 \\
\hline Food processing & 10.4 & 10.6 & 11.0 & 11.2 & 11.2 & 11.6 & 11.9 \\
\hline
\end{tabular}

Source: Goskomstat, and Fund staff estimates.

1/ Average wage fund data. 
Table 7. Russian Federation: Indicators of Hidden Unemployment, 1997-2003

\begin{tabular}{|c|c|c|c|}
\hline & \multicolumn{2}{|c|}{ Shortened Workday 1/ } & \multirow{2}{*}{$\begin{array}{l}\text { Forced Leave 2/ } \\
\text { Thousands } \\
\text { of persons }\end{array}$} \\
\hline & $\begin{array}{l}\text { Thousands } \\
\text { of persons }\end{array}$ & $\begin{array}{l}\text { In percent } \\
\text { of workforce }\end{array}$ & \\
\hline \multicolumn{4}{|l|}{1997} \\
\hline Q1 & 2,382 & 5.2 & 1,708 \\
\hline Q2 & 2,552 & 5.6 & 1,688 \\
\hline Q3 & 2,482 & 5.5 & 1,223 \\
\hline Q4 & 2,596 & 5.8 & 1,494 \\
\hline \multicolumn{4}{|l|}{1998} \\
\hline Q1 & 2,324 & 5.4 & 2,471 \\
\hline Q2 & 3,060 & 7.1 & 4,095 \\
\hline Q3 & 3,724 & 8.6 & 4,155 \\
\hline Q4 & 4,306 & 10.1 & 4,742 \\
\hline \multicolumn{4}{|l|}{1999} \\
\hline Q1 & 2,196 & 5.3 & 2,000 \\
\hline Q2 & 2,444 & 5.8 & 2,484 \\
\hline Q3 & 2,591 & 6.2 & 2,804 \\
\hline Q4 & 2,728 & 6.5 & 3,325 \\
\hline \multicolumn{4}{|l|}{2000} \\
\hline Q1 & 1,069 & 2.6 & 1,204 \\
\hline Q2 & 1,253 & 3.0 & 1,562 \\
\hline Q3 & 1,367 & 3.3 & 1,798 \\
\hline Q4 & 1,499 & 3.6 & 2,175 \\
\hline \multicolumn{4}{|l|}{2001} \\
\hline Q1 & 792 & 1.9 & 1,137 \\
\hline Q2 & 928 & 2.2 & 1,492 \\
\hline Q3 & 986 & 2.4 & 1,658 \\
\hline Q4 & 1,078 & 2.6 & 1,936 \\
\hline \multicolumn{4}{|l|}{2002} \\
\hline Q1 & 633 & 1.5 & 1,080 \\
\hline Q2 & 812 & 2.0 & 1,389 \\
\hline Q3 & 942 & 2.3 & 1,549 \\
\hline Q4 & 1,213 & 3.0 & 1,891 \\
\hline \multicolumn{4}{|l|}{2003} \\
\hline Q1 & 556 & 1.4 & 805 \\
\hline Q2 & 652 & 1.6 & 1,029 \\
\hline Q3 & 713 & 1.8 & 1,162 \\
\hline Q4 & 783 & 2.0 & 1,315 \\
\hline
\end{tabular}

Source: Goskomstat.

1/ For 1997, the data include the number of workers on shortened workdays at the end of each quarter; for 1998-2003 the data is year-to-date.

2 / Without pay or with partial pay. Cumulative data from the beginning of each year. 
Table 8. Russian Federation: Selected Labor Market Indicators, 1997-2003

\begin{tabular}{|c|c|c|c|c|}
\hline & \multirow[t]{2}{*}{ Employment 1/ } & \multirow{2}{*}{$\frac{\text { Unemployment 1/ }}{\text { ILO Definition }}$} & \multicolumn{2}{|c|}{ Registered Unemployment 2/ } \\
\hline & & & Total & $\begin{array}{c}\text { Receiving } \\
\text { Benefits }\end{array}$ \\
\hline & \multicolumn{4}{|c|}{ (In millions) } \\
\hline 1997 & 64.6 & 7.8 & 2.0 & 1.8 \\
\hline 1998 & 63.6 & 8.6 & 1.9 & 1.8 \\
\hline 1999 & 62.7 & 9.3 & 1.3 & 1.1 \\
\hline 2000 & 64.2 & 7.5 & 1.0 & 0.9 \\
\hline 2001 & 64.5 & 6.4 & 1.1 & 1.0 \\
\hline 2002 & 66.0 & 5.7 & 1.5 & 1.3 \\
\hline \multirow[t]{2}{*}{2003} & 65.7 & 6.0 & 1.6 & 1.3 \\
\hline & \multicolumn{4}{|c|}{ (In percent of labor force) } \\
\hline 1997 & 89.2 & 10.8 & 2.9 & 2.6 \\
\hline 1998 & 88.1 & 11.9 & 2.9 & 2.6 \\
\hline 1999 & 87.1 & 12.9 & 1.7 & 1.5 \\
\hline 2000 & 89.5 & 10.5 & 1.5 & 1.3 \\
\hline 2001 & 91.0 & 9.0 & 1.6 & 1.4 \\
\hline 2002 & 92.0 & 8.0 & 2.1 & 1.8 \\
\hline 2003 & 91.7 & 8.3 & 2.3 & 1.8 \\
\hline
\end{tabular}

Source: Goskomstat, and Ministry of Labour and Social Development.

1/ Annual averages, including small businesses.

2/ End-December data. 
Table 9. Russian Federation: Unemployment Rate by Regions (ILO methodology), 1997-2003 (In percent of labor force)

\begin{tabular}{|c|c|c|c|c|c|c|c|}
\hline & 1997 & 1998 & 1999 & 2000 & 2001 & 2002 & 2003 \\
\hline \multicolumn{8}{|l|}{ Northern Region } \\
\hline Karelian Republic & 11.9 & 16.6 & 15.7 & 11.5 & 8.7 & 7.9 & 8.5 \\
\hline Komi Republic & 13.9 & 17.6 & 16.1 & 12.1 & 14.0 & 9.1 & 11.9 \\
\hline Arkhangel'sk Oblast & 12.4 & 14.6 & 14.9 & 12.2 & 8.8 & 8.1 & 9.9 \\
\hline Nenets Autonomous Okrug & 13.3 & 11.5 & 20.0 & 10.9 & 7.2 & 7.2 & 8.6 \\
\hline Vologodsk Oblast & 10.5 & 12.4 & 11.5 & 8.0 & 8.9 & 6.0 & 4.8 \\
\hline Murmansk Oblast & 18.5 & 21.1 & 16.4 & 12.8 & 12.8 & 10.2 & 10.0 \\
\hline \multicolumn{8}{|l|}{ North-western region } \\
\hline Saint Petersburg & 9.9 & 11.3 & 11.0 & 6.3 & 3.9 & 3.4 & 4.1 \\
\hline Leningrad Oblast & 12.8 & 14.9 & 14.6 & 9.7 & 6.9 & 7.0 & 8.7 \\
\hline Novgorod Oblast & 13.5 & 14.8 & 14.1 & 7.8 & 6.4 & 6.3 & 5.0 \\
\hline Pskov Oblast & 14.2 & 15.9 & 13.3 & 12.5 & 10.3 & 8.2 & 8.1 \\
\hline \multicolumn{8}{|l|}{ Central region } \\
\hline Bryansk Oblast & 12.9 & 15.6 & 15.5 & 13.1 & 10.0 & 8.5 & 7.3 \\
\hline Vladimir Oblast & 11.6 & 12.1 & 12.1 & 12.2 & 9.9 & 10.3 & 10.1 \\
\hline Ivanovo Oblast & 16.9 & 18.6 & 17.5 & 10.1 & 5.6 & 6.8 & 6.5 \\
\hline Kaluzhska Oblast & 11.2 & 10.2 & 11.2 & 8.2 & 6.0 & 6.6 & 6.2 \\
\hline Kostromska Oblast & 9.4 & 11.0 & 10.0 & 8.6 & 6.0 & 5.0 & 6.3 \\
\hline Moscow & 4.8 & 4.8 & 5.6 & 3.8 & 2.1 & 1.4 & 1.3 \\
\hline Moscow Oblast & 8.8 & 9.9 & 10.5 & 7.4 & 5.5 & 4.3 & 4.3 \\
\hline Orlov Oblast & 9.8 & 12.6 & 8.6 & 8.5 & 7.9 & 6.7 & 7.8 \\
\hline Ryazan Oblast & 10.1 & 7.3 & 12.4 & 9.5 & 11.2 & 8.1 & 8.3 \\
\hline Smolensk Oblast & 12.9 & 16.4 & 13.6 & 12.2 & 9.9 & 11.1 & 10.9 \\
\hline Tver Oblast & 9.9 & 11.3 & 10.1 & 9.4 & 7.8 & 4.8 & 6.6 \\
\hline Tula Oblast & 10.0 & 11.6 & 11.3 & 9.7 & 5.2 & 6.1 & 5.3 \\
\hline Yaroslavl Oblast & 8.8 & 11.2 & 8.7 & 7.3 & 7.1 & 3.8 & 5.7 \\
\hline \multicolumn{8}{|l|}{ Volga region } \\
\hline Marii-El Republic & 18.0 & 13.1 & 10.3 & 11.3 & 9.4 & 13.8 & 12.2 \\
\hline Mordoviya Republic & 12.2 & 13.7 & 12.6 & 10.7 & 10.5 & 9.4 & 7.4 \\
\hline Chuvash Republic & 13.9 & 13.6 & 13.1 & 9.1 & 9.6 & 9.5 & 8.6 \\
\hline Kirov Oblast & 11.4 & 13.1 & 9.5 & 8.2 & 7.7 & 6.9 & 7.4 \\
\hline Nizhegorod Oblast & 9.7 & 9.2 & 7.4 & 7.5 & 8.3 & 7.8 & 6.1 \\
\hline \multicolumn{8}{|l|}{ Central-Chernozem region } \\
\hline Belgorod Oblast & 10.7 & 11.2 & 12.2 & 5.8 & 6.5 & 8.2 & 8.2 \\
\hline Voronezh Oblast & 8.1 & 9.7 & 11.4 & 10.0 & 9.6 & 8.9 & 8.1 \\
\hline Kursk Oblast & 8.1 & 10.3 & 10.7 & 10.4 & 10.4 & 7.3 & 8.6 \\
\hline Lipetsk Oblast & 9.8 & 10.9 & 10.3 & 8.5 & 6.6 & 4.9 & 4.4 \\
\hline Tambov Oblast & 12.9 & 12.6 & 12.8 & 8.2 & 12.4 & 9.7 & 9.1 \\
\hline \multicolumn{8}{|l|}{ Povolgski region } \\
\hline Kalmykiya Republic & 26.1 & 31.3 & 23.8 & 20.1 & 19.1 & 18.1 & 17.9 \\
\hline Tatarstan Republic & 7.9 & 11.1 & 11.1 & 8.0 & 6.3 & 5.3 & 6.7 \\
\hline Astrakhan Oblast & 14.6 & 15.8 & 13.9 & 11.5 & 10.5 & 11.1 & 10.1 \\
\hline Volgograd Oblast & 14.4 & 14.1 & 11.8 & 9.7 & 9.8 & 8.4 & 10.9 \\
\hline Penzensk Oblast & 12.0 & 17.3 & 10.8 & 11.2 & 13.9 & 7.4 & 9.1 \\
\hline Samara Oblast & 9.3 & 8.7 & 12.2 & 10.3 & 6.0 & 5.4 & 4.4 \\
\hline Saratov Oblast & 15.8 & 15.9 & 10.7 & 9.5 & 10.0 & 7.5 & 10.5 \\
\hline Ulyanov Oblast & 9.8 & 11.1 & 8.8 & 6.4 & 9.0 & 6.1 & 7.4 \\
\hline
\end{tabular}

Continued on next page. 
Table 9 (contd). Russian Federation: Unemployment Rate by Regions (ILO methodology), 1997-2003 (In percent of labor force)

\begin{tabular}{|c|c|c|c|c|c|c|c|}
\hline & 1997 & 1998 & 1999 & 2000 & 2001 & 2002 & 2003 \\
\hline \multicolumn{8}{|l|}{ North-Kaukaz region } \\
\hline Adygeya Republic & 12.3 & 15.6 & 19.6 & 14.1 & 14.1 & 13.5 & 15.8 \\
\hline Dagestan Republic & 27.0 & 30.0 & 27.9 & 25.6 & 28.8 & 23.9 & 20.1 \\
\hline Ingush Republic & 58.2 & 50.9 & 51.8 & 32.0 & 34.9 & 44.0 & 53.1 \\
\hline Kabardino-Balkar Republic & 17.7 & 23.9 & 25.1 & 16.6 & 16.8 & 18.7 & 22 \\
\hline Karachaev-Circassian Republic & 18.9 & 25.5 & 21.4 & 20.7 & 18.6 & 12.0 & 19 \\
\hline North Ossetian-Alaniya Republic & 22.2 & 26.6 & 32.0 & 28.5 & 16.7 & 12.5 & 10.1 \\
\hline \multicolumn{8}{|l|}{ Chechen Republic } \\
\hline Krasnodarsk Krai & 16.5 & 16.3 & 15.2 & 12.5 & 10.7 & 7.6 & 10.1 \\
\hline Stavropol Krai & 13.9 & 16.2 & 18.5 & 13.8 & 9.8 & 9.6 & 10.3 \\
\hline Rostov Oblast & 12.0 & 15.6 & 17.8 & 14.9 & 12.9 & 11.3 & 12.3 \\
\hline \multicolumn{8}{|l|}{ Ural } \\
\hline Bashkortostan Republic & 11.2 & 13.3 & 12.1 & 11.5 & 10.7 & 8.3 & 8.2 \\
\hline Udmurt Republic & 12.1 & 12.6 & 11.3 & 9.3 & 7.5 & 7.9 & 6.7 \\
\hline Kurgan Oblast & 12.5 & 12.9 & 12.6 & 13.2 & 13.3 & 10.7 & 9.6 \\
\hline Orenburg Oblast & 9.5 & 12.7 & 13.6 & 11.8 & 8.5 & 10.3 & 11.3 \\
\hline Perm Oblast & 11.1 & 12.7 & 13.9 & 10.4 & 6.9 & 9.1 & 6.9 \\
\hline Komi-Permyatsk Autonomous Okrug & 17.6 & 17.1 & 8.3 & 8.0 & 12.2 & 12.5 & 10.9 \\
\hline Sverdlovsk Oblast & 10.2 & 10.5 & 13.9 & 10.0 & 7.7 & 8.4 & 7.6 \\
\hline Chelyabinsk Oblast & 9.5 & 12.4 & 11.8 & 8.1 & 8.7 & 6.4 & 5.9 \\
\hline \multicolumn{8}{|l|}{ West-Siberia } \\
\hline Altai Republic & 18.4 & 18.0 & 19.0 & 17.5 & 9.7 & 11.5 & 10.2 \\
\hline Altai Krai & 13.9 & 16.1 & 12.5 & 11.5 & 9.9 & 8.3 & 12.1 \\
\hline Kemerovo Oblast & 11.2 & 12.4 & 13.7 & 10.4 & 12.4 & 9.3 & 9.7 \\
\hline Novosibirsk Oblast & 10.7 & 13.5 & 14.5 & 13.4 & 10.0 & 11.4 & 11 \\
\hline Omsk Oblast & 13.4 & 15.4 & 14.5 & 13.9 & 10.0 & 9.5 & 9.5 \\
\hline Tomsk Oblast & 12.8 & 14.7 & 16.2 & 12.2 & 9.8 & 11.1 & 13.7 \\
\hline Tyumen Oblast & 8.9 & 14.0 & 11.1 & 10.3 & 10.4 & 8.7 & 8.3 \\
\hline Khanti-Mansi Autonomous Okrug & 12.5 & 14.6 & 11.3 & 11.2 & 11.0 & 10.2 & 9.4 \\
\hline Yamalo-Nenetsk Autonomous Okrug & 10.7 & 11.2 & 10.0 & 7.9 & 7.1 & 6.9 & 5.5 \\
\hline \multicolumn{8}{|l|}{ East Siberia } \\
\hline Buryat Republic & 21.3 & 22.0 & 17.3 & 19.1 & 18.5 & 15.4 & 16.8 \\
\hline Tyva Republic & 22.0 & 20.9 & 25.7 & 22.9 & 23.9 & 20.5 & 20.7 \\
\hline Khakasian Republic & 13.0 & 9.6 & 15.7 & 12.3 & 8.8 & 8.0 & 10.5 \\
\hline Krasnoyarsk Krai & 13.3 & 16.4 & 14.2 & 11.9 & 9.7 & 7.7 & 11.2 \\
\hline Taimyrsk Autonomous Okrug & 7.0 & 15.5 & 9.6 & 5.7 & 7.3 & 7.6 & 6.9 \\
\hline Evenkisk Autonomous Okrug & 3.4 & 6.2 & 6.9 & 3.2 & 2.9 & 2.5 & 5 \\
\hline Irkutsk Oblast & 14.4 & 13.6 & 14.7 & 11.4 & 10.9 & 11.0 & 11.7 \\
\hline Ust-Ordinsk Buryat Autonomous Okrug & 7.7 & 8.2 & 11.8 & 8.9 & 14.2 & 12.2 & 10.9 \\
\hline Chitinsk Oblast & 18.5 & 19.8 & 20.1 & 13.9 & 17.0 & 11.1 & 15.3 \\
\hline Aginsk Buryat A. Okrug & 28.1 & 35.2 & 20.5 & 25.6 & 23.0 & 25.3 & 12.9 \\
\hline \multicolumn{8}{|l|}{ Far East region } \\
\hline Sakha republic (Yakutiya) & 12.6 & 13.4 & 13.8 & 11.3 & 8.2 & 7.1 & 9.4 \\
\hline Jewish Autonomous Oblast & 25.1 & 23.9 & 17.6 & 15.2 & 9.5 & 9.1 & 6.5 \\
\hline Chukotsk A. Oblast & 8.4 & 4.7 & 9.3 & 10.0 & 7.4 & 4.6 & 4.8 \\
\hline Primorye Krai & 13.3 & 14.7 & 13.5 & 11.9 & 8.6 & 8.9 & 7.9 \\
\hline Khabarovsk Krai & 12.7 & 12.5 & 14.2 & 11.6 & 10.3 & 6.7 & 6.1 \\
\hline Amur Oblast & 15.6 & 16.2 & 16.2 & 13.4 & 12.2 & 11.0 & 10.1 \\
\hline Kamchatka Oblast & 12.5 & 17.8 & 18.1 & 16.0 & 14.5 & 11.6 & 11.4 \\
\hline Koryak Autonomous Okrug & 6.8 & 8.4 & 8.8 & 12.5 & 15.2 & 8.8 & 13.6 \\
\hline Magadan Oblast & 13.6 & 17.9 & 20.3 & 10.8 & 11.7 & 8.2 & 10.2 \\
\hline Sakhalin Oblast & 15.0 & 17.4 & 20.4 & 13.0 & 12.0 & 9.5 & 9.1 \\
\hline Kaliningrad Oblast & 11.5 & 16.8 & 15.6 & 15.4 & 9.6 & 7.1 & 7.5 \\
\hline
\end{tabular}

\footnotetext{
Source: Goskomstat.
} 
Table 10. Russian Federation: Regional Migration, 1997-2003 1/ (In thousands)

\begin{tabular}{|c|c|c|c|c|c|c|c|}
\hline & 1997 & 1998 & 1999 & 2000 & 2001 & 2002 & 2003 \\
\hline \multicolumn{8}{|l|}{ Federal districts: } \\
\hline Central & 80.8 & 85.4 & 82.9 & 73.9 & 72.6 & 81.3 & 90.4 \\
\hline Belgorod Oblast & 6.8 & 7.6 & 9.4 & 6.9 & 6.5 & 7.1 & 6.9 \\
\hline Bryansk Oblast & 0.4 & 0.9 & 1.0 & -0.3 & -0.6 & -0.6 & -0.8 \\
\hline Vladimir Oblast & 2.9 & 2.1 & 1.1 & 0.5 & 1.0 & 0.5 & -0.4 \\
\hline Voronezh Oblast & 1.1 & 3.8 & 3.8 & 1.4 & $\ldots$ & 1.5 & 1.7 \\
\hline Ivanovo Oblast & 0.9 & 1.1 & 1.4 & 0.7 & 0.1 & -0.2 & -0.6 \\
\hline Kaluzhska Oblast & 1.0 & 0.3 & -0.9 & -1.3 & -0.7 & -1.6 & -1.9 \\
\hline Kostromska Oblast & 1.0 & 1.0 & 1.0 & 1.0 & 0.1 & 0.3 & -0.1 \\
\hline Kursk Oblast & 0.4 & -0.9 & -1.0 & -2.3 & -2.9 & -3.1 & -3.9 \\
\hline Lipetsk Oblast & 3.2 & 1.9 & 2.6 & 1.8 & 1.6 & 0.2 & -0.4 \\
\hline Moscow Oblast & 24.2 & 22.6 & 18.0 & 22.7 & 30.3 & 40.6 & 49.9 \\
\hline Orlov Oblast & 0.6 & 0.7 & 1.1 & 0.1 & 0.7 & -0.1 & -0.4 \\
\hline Ryazan Oblast & -0.8 & -1.2 & -0.4 & -1.4 & -1.3 & -1.2 & -1.4 \\
\hline Smolensk Oblast & -0.4 & -0.9 & -1.2 & -2.0 & -1.9 & -1.3 & -0.9 \\
\hline Tambov Oblast & -1.1 & -1.3 & -1.1 & -2.1 & -3.3 & -3.2 & -2.8 \\
\hline Tver Oblast & 1.4 & 0.2 & -0.4 & -2.0 & -1.9 & -0.9 & -1.8 \\
\hline Tula Oblast & 0.3 & -0.5 & -0.7 & -2.0 & -2.4 & -1.6 & -1.8 \\
\hline Yaroslavl Oblast & 2.3 & 1.5 & 2.0 & 1.0 & 0.8 & 1.8 & 1.1 \\
\hline Moscow & 36.6 & 46.7 & 47.2 & 51.3 & 46.4 & 43.0 & 47.9 \\
\hline North West & -13.1 & -8.4 & -12.6 & -1.6 & 6.6 & 4.0 & 2.4 \\
\hline Karelian Republic & -0.2 & -0.4 & -0.3 & 0.6 & 0.6 & 0.8 & 0.2 \\
\hline Komi Republic & -11.1 & -10.5 & -10.7 & -6.7 & -5.0 & -6.3 & -5.8 \\
\hline Arkhangel'sk Oblast & -7.9 & -7.9 & -8.1 & -6.4 & -5.1 & -5.0 & -5.5 \\
\hline Nenets Autonomous Okrug & -0.8 & -0.5 & -0.3 & -0.2 & $\ldots$ & 0.2 & 0.0 \\
\hline Vologodsk Oblast & 1.5 & 1.3 & 0.9 & 0.6 & -0.2 & -0.1 & 0.1 \\
\hline Kaliningrad Oblast & 4.4 & 4.1 & 0.2 & 0.7 & 1.9 & 2.3 & 2.3 \\
\hline Leningrad Oblast & 11.2 & 9.1 & 9.3 & 10.0 & 11.0 & 11.9 & 13.1 \\
\hline Murmansk Oblast & -16.1 & -16.3 & -13.6 & -9.9 & -7.6 & -8.0 & -6.5 \\
\hline Novgorod Oblast & 3.0 & 1.9 & 1.0 & 0.2 & $\ldots$ & 0.5 & -0.4 \\
\hline Pskov Oblast & 1.0 & 0.2 & 0.7 & $\ldots$ & -0.2 & 0.4 & 0.9 \\
\hline Saint Petersburg & 1.0 & 10.2 & 8.1 & 9.3 & 11.2 & 7.5 & 4.0 \\
\hline South & 3.2 & -3.7 & -0.5 & -12.6 & -2.4 & -4.5 & -5.9 \\
\hline Adygeya Republic & 1.4 & 0.6 & 0.3 & -0.1 & 0.6 & 1.2 & 0.5 \\
\hline Dagestan Republic & -5.6 & -1.4 & -1.1 & -5.7 & -5.4 & -5.3 & -5.6 \\
\hline $\begin{array}{l}\text { Ingush Republic } \\
\text { Chechen Republic }\end{array}$ & -21.3 & -20.5 & -19.3 & -11.7 & -0.7 & 0.8 & 1.6 \\
\hline Kabardino-Balkar Republic & -0.5 & -1.2 & -1.6 & -1.9 & -1.9 & -2.0 & -2.1 \\
\hline Kalmykiya Republic & -1.4 & -1.5 & -1.6 & -2.2 & -2.1 & -1.3 & -1.5 \\
\hline Karachaev-Circassian Republic & -0.9 & -1.0 & -2.1 & -2.0 & -2.2 & -2.3 & -2.1 \\
\hline North Ossetian-Alaniya Republic & -3.0 & -2.1 & -2.5 & -2.4 & -1.9 & -2.4 & -2.7 \\
\hline Krasnodarsk Krai & 16.2 & 10.7 & 19.3 & 12.6 & 13.7 & 8.4 & 10.4 \\
\hline Stavropol Krai & 11.4 & 9.2 & 7.5 & 2.1 & 0.7 & 2.4 & 2.5 \\
\hline Astrakhan Oblast & -0.3 & -0.5 & -0.3 & 0.7 & -0.5 & -0.5 & -0.8 \\
\hline Volgograd Oblast & 5.3 & 3.2 & 0.3 & -2.7 & -2.6 & -2.9 & -3.2 \\
\hline Rostov Oblast & 2.0 & 0.9 & 0.6 & 0.5 & -0.2 & -0.7 & -2.9 \\
\hline
\end{tabular}

Continued on next page.

1/ A positive entry indicates a net inflow. 
Table 10 (contd.) Russian Federation: Regional Migration, 1997-2003 1/ (In thousands)

\begin{tabular}{|c|c|c|c|c|c|c|c|}
\hline & 1997 & 1998 & 1999 & 2000 & 2001 & 2002 & 2003 \\
\hline Volga & 18.2 & 20.5 & 23.3 & -2.3 & -13.5 & -14.1 & -21.4 \\
\hline Bashkortostan Republic & 1.6 & 2.5 & 4.9 & -2.0 & -3.5 & -2.4 & -2.8 \\
\hline Marii-El Republic & 0.2 & 0.4 & 1.1 & 0.0 & -0.6 & -0.5 & -0.6 \\
\hline Mordoviya Republic & -1.8 & -1.9 & -1.6 & -2.3 & -2.9 & -2.8 & -2.6 \\
\hline Tatarstan Republic & 6.6 & 5.5 & 4.5 & 3.0 & 3.2 & 3.2 & 3.6 \\
\hline Udmurt Republic & 1.3 & 1.3 & 0.3 & -0.8 & -1.6 & -1.7 & -1.5 \\
\hline Chuvash Republic & 1.3 & 2.4 & 2.0 & 0.5 & -0.3 & -0.3 & 0.0 \\
\hline Kirov Oblast & -2.2 & -1.9 & -1.0 & -2.1 & -2.9 & -3.1 & -4.2 \\
\hline Nizhegorod Oblast & 9.0 & 7.8 & 6.4 & 2.8 & 1.6 & 1.5 & 0.4 \\
\hline Orenburg Oblast & -2.8 & -3.9 & -3.0 & -4.4 & -4.4 & -5.2 & -4.6 \\
\hline Penzensk Oblast & 0.6 & 0.3 & 0.0 & -1.3 & -1.5 & -1.6 & -1.7 \\
\hline Perm Oblast & -0.3 & 0.4 & 2.0 & 0.7 & -0.2 & -0.5 & -3.4 \\
\hline Komi-Permyatsk Autonomous Okrug & -1.3 & -0.5 & -0.3 & -0.3 & -0.2 & 0.0 & -0.1 \\
\hline Samara Oblast & 6.5 & 7.2 & 7.1 & 5.4 & 3.5 & 3.7 & 0.9 \\
\hline Saratov Oblast & 0.9 & 3.0 & 2.4 & 0.6 & -0.5 & -0.7 & -1.6 \\
\hline Ulyanov Oblast & -2.8 & -2.5 & -1.7 & -2.3 & -3.4 & -3.6 & -3.4 \\
\hline Urals & 5.0 & -4.7 & -14.3 & 1.6 & 3.5 & -2.4 & -4.7 \\
\hline Kurgan Oblast & -3.6 & -2.7 & -2.3 & -3.9 & -5.6 & -4.5 & -4.8 \\
\hline Sverdlovsk Oblast & 2.1 & 1.3 & 1.4 & 1.0 & -0.7 & -1.7 & 0.1 \\
\hline Tyumen Oblast & 2.0 & -7.6 & -15.1 & 4.4 & 10.6 & 7.1 & 3.4 \\
\hline Khanti-Mansi Autonomous Okrug & 4.2 & -2.6 & -8.8 & 9.4 & 11.4 & 7.0 & 4.2 \\
\hline Yamalo-Nenetsk Autonomous Okrug & -2.0 & -5.0 & -6.3 & -2.6 & -0.4 & 0.2 & 0.3 \\
\hline Chelyabinsk Oblast & 4.6 & 4.3 & 1.6 & 0.1 & -0.9 & -3.2 & -3.4 \\
\hline Siberian & -30.5 & -25.7 & -21.6 & -22.4 & -26.1 & -27.7 & -27.7 \\
\hline Altai Republic & 0.3 & 0.4 & 0.3 & 0.2 & -0.3 & 0.0 & -0.1 \\
\hline Buryat Republic & -5.4 & -5.3 & -4.7 & -4.2 & -5.0 & -4.4 & -3.6 \\
\hline Tyva Republic & -1.0 & -0.7 & -0.7 & -1.0 & -1.2 & -0.8 & -0.7 \\
\hline Khakasian Republic & 1.3 & 0.9 & 1.3 & 1.2 & 0.6 & 0.5 & 1.1 \\
\hline Altai Krai & -2.1 & -3.2 & -0.6 & -2.9 & -5.5 & -3.7 & -4.5 \\
\hline Krasnoyarsk Krai & -9.5 & -8.7 & -9.4 & -4.9 & -2.2 & -4.1 & -5.0 \\
\hline Taimyrsk Autonomous Okrug & -1.3 & -1.1 & -0.7 & -0.1 & 0.3 & -0.1 & -0.4 \\
\hline Evenkisk Autonomous Okrug & -0.4 & -0.5 & -0.5 & -0.4 & -0.1 & -0.1 & -0.1 \\
\hline Irkutsk Oblast & -6.6 & -4.4 & -3.5 & -1.6 & -3.8 & -4.4 & -4.9 \\
\hline Ust-Ordinsk Buryat Autonomous Okrug & 0.2 & 0.1 & 0.1 & -0.4 & -0.1 & -0.2 & -0.2 \\
\hline Kemerovo Oblast & -4.6 & -2.4 & 0.0 & -0.1 & 0.1 & -0.1 & 0.8 \\
\hline Novosibirsk Oblast & 6.0 & 6.9 & 4.1 & 0.8 & 1.2 & -0.9 & -2.1 \\
\hline Omsk Oblast & 0.1 & -0.2 & -1.2 & -3.4 & -3.7 & -3.4 & -2.8 \\
\hline Tomsk Oblast & -0.3 & -0.5 & -1.2 & -0.1 & -0.1 & 0.2 & -0.9 \\
\hline Chitinsk Oblast & -8.8 & -8.6 & -6.2 & -6.6 & -6.2 & -6.5 & -5.0 \\
\hline Aginsk Buryat A. Okrug & -0.6 & -0.2 & -0.3 & -0.3 & -0.1 & -0.1 & 0.1 \\
\hline Far East & -63.6 & -63.3 & -57.0 & -36.6 & -31.2 & -27.4 & -24.3 \\
\hline Sakha republic (Yakutiya) & -16.4 & -18.1 & -14.2 & -7.2 & -7.0 & -5.8 & -4.5 \\
\hline Primorye Krai & -8.3 & -7.8 & -8.7 & -6.1 & -7.3 & -5.6 & -5.7 \\
\hline Khabarovsk Krai & -4.8 & -5.4 & -5.8 & -1.9 & -1.5 & -1.0 & 0.7 \\
\hline Amur Oblast & -5.5 & -5.9 & -5.1 & -3.9 & -3.4 & -3.3 & -2.8 \\
\hline Kamchatka Oblast & -7.2 & -6.6 & -6.3 & -4.4 & -3.1 & -2.9 & -2.7 \\
\hline Koryak Autonomous Okrug & -1.0 & -0.9 & -0.8 & -0.7 & -0.3 & -0.3 & -0.2 \\
\hline Magadan Oblast & -6.4 & -6.1 & -6.3 & -5.2 & -3.9 & -3.3 & -3.3 \\
\hline Sakhalin Oblast & -11.3 & -9.8 & -7.1 & -4.5 & -3.6 & -3.7 & -4.0 \\
\hline Jewish Autonomous Oblast & $\ldots$ & $\ldots$ & 0.1 & -0.2 & 0.1 & 0.0 & -0.2 \\
\hline Chukotsk A. Oblast & -3.7 & -3.6 & -3.5 & -3.1 & -1.5 & -1.7 & -1.9 \\
\hline
\end{tabular}

Source: Goskomstat.

1/ A positive entry indicates a net inflow. 
Table 11. Russian Federation: Consumer Price Inflation and CPI Weights, 1997-2003

\begin{tabular}{rccrr}
\hline & $\begin{array}{c}\text { Overall } \\
\text { CPI }\end{array}$ & Food 1/ & Nonfood 2/ & $\begin{array}{c}\text { Paid } \\
\text { Services 3/ }\end{array}$ \\
\hline \multicolumn{4}{c}{ (Percent change, end of period) } \\
1997 & 11.4 & 9.9 & 8.1 & 22.5 \\
1998 & 84.4 & 96.0 & 99.5 & 18.3 \\
1999 & 36.5 & 35.9 & 39.2 & 34.0 \\
2000 & 20.2 & 17.9 & 18.5 & 33.7 \\
2001 & 18.6 & 17.1 & 12.7 & 36.9 \\
2002 & 15.1 & 11.3 & 10.9 & 36.2 \\
2003 & 12.0 & 10.2 & 9.2 & 22.3 \\
& & & & \\
1997 & 100 & 54.4 & 29.0 & 16.6 \\
1998 & 100 & 51.9 & 32.1 & 16.0 \\
1999 & 100 & 59.9 & 27.2 & 12.9 \\
2000 & 100 & 58.7 & 27.9 & 13.4 \\
2001 & 100 & 55.3 & 30.6 & 14.1 \\
2002 & 100 & 53.2 & 30.5 & 16.3 \\
2003 & 100 & 51.6 & 30.9 & 17.5 \\
\hline
\end{tabular}

Source: Goskomstat.

1/ Includes food and beverages; excludes tobacco.

2/ Includes clothing and footwear, household goods, medicine, recreation, education, culture, and personal care and effects.

3/ Includes rent, water, fuel and power. 


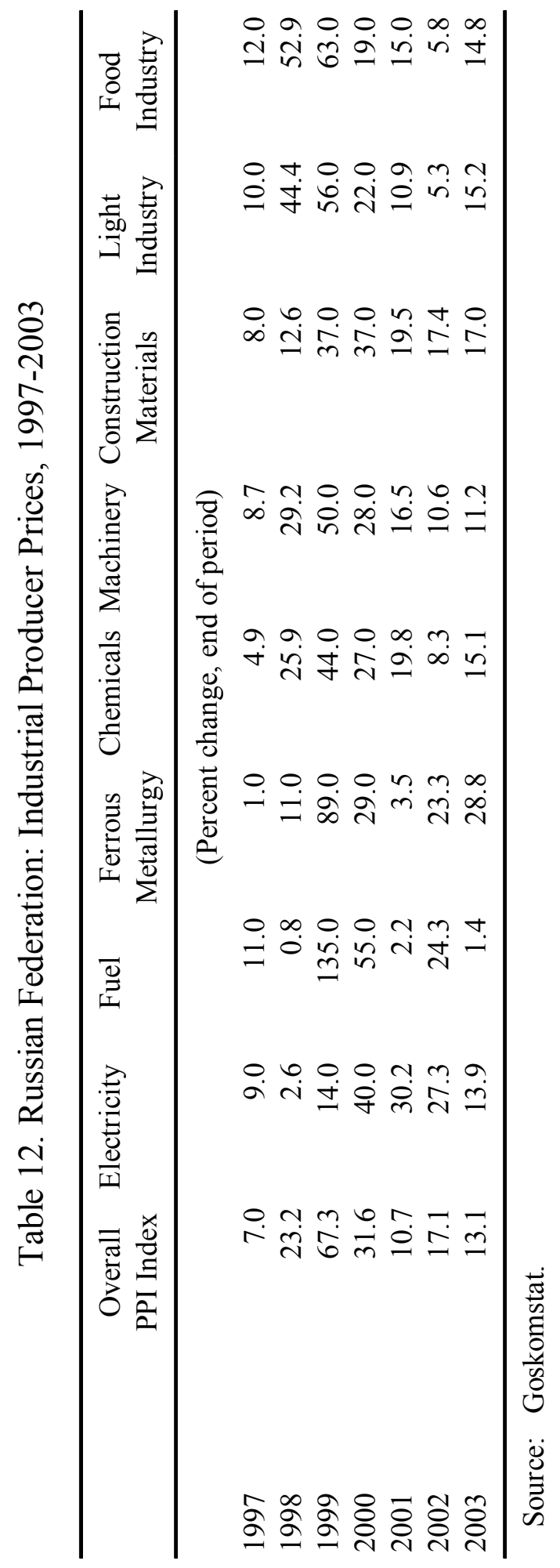


Table 13. Russian Federation: Wages, Pensions, and Per Capita Income, 1997-2003

\begin{tabular}{lrrrrrrr}
\hline & 1997 & 1998 & 1999 & 2000 & 2001 & 2002 & 2003 \\
\hline & \multicolumn{2}{c}{ (In rubles per month, annual average unless otherwise indicated) } \\
& 965 & 1,108 & 1,581 & 2,253 & 3,283 & 4,415 & 5,510 \\
Nominal wage & 83 & 83 & 83 & 132 & 300 & 450 & 600 \\
Minimum wage 1/ & 366 & 403 & 522 & 823 & 1,138 & 1,462 & 1,747 \\
Pensions & 942 & 1,013 & 1,663 & 2,288 & 3,075 & 3,964 & 5,142 \\
Income per capita & & & & & & \\
& & & & & & \\
& 4.4 & -10.0 & -23.2 & 18.0 & 20.0 & 16.2 & 9.8 \\
Real wage & -0.9 & -45.8 & -26.8 & 31.6 & 91.7 & 30.4 & 19.1 \\
Real minimum wage 1/ & 3.0 & -40.0 & -5.0 & 31.0 & 17.0 & 12.0 & 7.0 \\
Real pensions & 6.4 & -16.0 & -11.9 & 13.4 & 10.0 & 10.7 & 13.6 \\
Real income per capita & & & & & & \\
\hline
\end{tabular}

Sources: Goskomstat, Ministry of Labor, Pension Fund, and Fund staff estimates.

$1 /$ Data at end of year. 
Table 14. Russian Federation: Summary Operations of the Enlarged Government, 1997-2003 1/ (In billions of rubles, unless otherwise indicated)

\begin{tabular}{|c|c|c|c|c|c|c|c|}
\hline & 1997 & 1998 & 1999 & 2000 & 2001 & 2002 & 2003 \\
\hline Federal government balance & -180 & -158 & -204 & 62 & 242 & 140 & 207 \\
\hline Federal government primary balance & -62 & -37 & 83 & 379 & 484 & 367 & 431 \\
\hline Revenues & 310 & 299 & 608 & 1,128 & 1,591 & 1,861 & 2,218 \\
\hline Expenditures & 490 & 457 & 812 & 1,066 & 1,349 & 1,721 & 2,011 \\
\hline of which: interest & 118 & 122 & 288 & 317 & 242 & 227 & 224 \\
\hline Local government balance & -22 & -33 & -1 & 62 & -22 & -53 & -70 \\
\hline Revenues & 467 & 436 & 719 & 1,129 & 1,342 & 1,644 & 1,929 \\
\hline of which: transfers & 85 & 53 & 81 & 123 & 242 & 293 & 322 \\
\hline Expenditures & 489 & 469 & 721 & 1,068 & 1,364 & 1,697 & 1,999 \\
\hline Extrabudgetary funds balance & 3 & -24 & 56 & 106 & 22 & -23 & 8 \\
\hline Revenues & 251 & 234 & 391 & 604 & 713 & 956 & 1,134 \\
\hline of which: federal transfers & 23 & 12 & 18 & 43 & 64 & 93 & 99 \\
\hline Expenditures & 248 & 258 & 335 & 498 & 691 & 979 & 1,126 \\
\hline Enlarged government balance (-deficit) & -199 & -215 & -150 & 229 & 242 & 63 & 145 \\
\hline Enlarged government primary balance & -81 & -94 & 138 & 546 & 484 & 290 & 369 \\
\hline Revenues 2/ & 920 & 903 & 1,619 & 2,694 & 3,339 & 4,075 & 4,861 \\
\hline Expenditures 2/ & 1,119 & 1,119 & 1,769 & 2,466 & 3,097 & 4,012 & 4,716 \\
\hline Financing of the enlarged government & 199 & 215 & 150 & -229 & -242 & -63 & -141 \\
\hline Foreign & 40 & 56 & 9 & -89 & -171 & -144 & -230 \\
\hline Domestic & 148 & 92 & 29 & -211 & -76 & 80 & 74 \\
\hline Monetary authorities & 30 & 127 & 29 & -242 & -77 & -34 & -156 \\
\hline Commerical banks & 14 & -68 & 13 & 43 & 13 & 85 & 60 \\
\hline Other & 104 & 34 & -13 & -11 & -13 & 28 & 169 \\
\hline Arrears and rescheduling & 10 & 68 & 112 & 70 & 5 & 1 & 15 \\
\hline Domestic expenditure & 10 & 52 & -13 & -65 & 4 & 1 & 15 \\
\hline \multirow[t]{2}{*}{ Foreign interest } & 0 & 15 & 125 & 135 & 0 & 0 & 0 \\
\hline & \multicolumn{7}{|c|}{ (In percent of GDP) } \\
\hline Federal govt overall balance & -7.7 & -6.0 & -4.2 & 0.8 & 2.7 & 1.3 & 1.5 \\
\hline Federal govt primary balance & -2.6 & -1.4 & 1.7 & 5.2 & 5.4 & 3.4 & 3.2 \\
\hline Revenues & 13.3 & 11.4 & 12.6 & 15.4 & 17.8 & 17.2 & 16.7 \\
\hline Expenditures & 20.9 & 17.4 & 16.8 & 14.6 & 15.1 & 15.9 & 15.1 \\
\hline Local govt overall balance & -0.9 & -1.2 & 0.0 & 0.8 & -0.2 & -0.5 & -0.5 \\
\hline Revenues & 19.9 & 16.6 & 14.9 & 15.5 & 15.0 & 15.2 & 14.5 \\
\hline of which: transfers & 3.6 & 2.0 & 1.7 & 1.7 & 2.7 & 2.7 & 2.4 \\
\hline Expenditures & 20.9 & 17.8 & 14.9 & 14.6 & 15.2 & 15.7 & 15.0 \\
\hline Extrabudgetary funds balance & 0.1 & -0.9 & 1.2 & 1.4 & 0.2 & -0.2 & 0.1 \\
\hline Revenues & 10.7 & 8.9 & 8.1 & 8.3 & 8.0 & 8.8 & 8.5 \\
\hline of which: transfers & 1.0 & 0.5 & 0.4 & 0.6 & 0.7 & 0.9 & 0.7 \\
\hline Expenditures & 10.6 & 9.8 & 6.9 & 6.8 & 7.7 & 9.0 & 8.5 \\
\hline Enlarged government overall balance & -8.5 & -8.2 & -3.1 & 3.1 & 2.7 & 0.6 & 1.1 \\
\hline Enlarged government primary balance & -3.5 & -3.6 & 2.9 & 7.5 & 5.4 & 2.7 & 2.7 \\
\hline Revenues 2/ & 39.3 & 34.4 & 33.6 & 36.9 & 37.3 & 37.6 & 36.6 \\
\hline Expenditures 2/ & 47.8 & 42.5 & 36.7 & 33.7 & 34.6 & 37.0 & 35.5 \\
\hline
\end{tabular}

Sources: Ministry of Finance, CBR, Goskomstat.

1/ On a commitment basis. In 1997, includes wage arrears, arrears in transfers to the Pension Fund, and accumulation of all federal spending arrears. In 1998, includes local wage and pension arrears. In 1999, includes accumulation of civilian arrears.

2/ Consolidated revenues and expenditures (excluding intragovernmental transfers) and including both cash and noncash items. 
Table 15. Russian Federation: Federal Government Budget Execution, 1997-2003 (In billions of rubles, unless otherwise indicated)

\begin{tabular}{|c|c|c|c|c|c|c|c|}
\hline & 1997 & 1998 & 1999 & 2000 & 2001 & 2002 & 2003 \\
\hline Revenue & 310 & 299 & 608 & 1,128 & 1,591 & 1,861 & 2,218 \\
\hline Cash revenue & 252 & 243 & 608 & 1,128 & 1,591 & 1,861 & 2,218 \\
\hline Noncash revenue 1 / & 59 & 56 & 0 & 0 & 0 & 0 & 0 \\
\hline Tax Revenue & 261 & 253 & 505 & 965 & 1,461 & 1,696 & 2,030 \\
\hline Profit and personal income taxes & 35 & 37 & 99 & 206 & 218 & 173 & 173 \\
\hline VAT & 117 & 117 & 219 & 372 & 639 & 753 & 882 \\
\hline Excises & 50 & 57 & 84 & 131 & 203 & 215 & 253 \\
\hline Taxes on trade & 28 & 34 & 86 & 229 & 329 & 323 & 453 \\
\hline Import tariffs & 28 & 34 & 47 & 65 & 104 & 129 & 158 \\
\hline Export taxes & 0 & 0 & 39 & 164 & 225 & 195 & 295 \\
\hline Other (incl. natural resource taxes) & 31 & 8 & 17 & 27 & 70 & 232 & 269 \\
\hline Non-tax revenue & 11 & 19 & 48 & 70 & 115 & 150 & 174 \\
\hline Budgetary funds & 38 & 26 & 55 & 93 & 15 & 15 & 14 \\
\hline Expenditure & 490 & 457 & 812 & 1,066 & 1,349 & 1,721 & 2,011 \\
\hline Non-interest expenditure 1/ & 372 & 335 & 525 & 749 & 1,107 & 1,494 & 1,786 \\
\hline Government administration & 10 & 10 & 15 & 25 & 42 & 56 & 67 \\
\hline International activity & 4 & 9 & 36 & 37 & 75 & 40 & 31 \\
\hline Defense & 80 & 61 & 116 & 191 & 247 & 295 & 356 \\
\hline Law enforcement and justice & 44 & 36 & 60 & 114 & 161 & 210 & 273 \\
\hline Sectoral expenditures & 55 & 26 & 41 & 72 & 134 & 161 & 160 \\
\hline Education & 14 & 14 & 21 & 38 & 54 & 82 & 100 \\
\hline Health and emergency management & 15 & 13 & 18 & 26 & 30 & 40 & 63 \\
\hline Social policy & 23 & 37 & 49 & 66 & 112 & 153 & 135 \\
\hline Culture and mass media & 3 & 2 & 5 & 11 & 14 & 20 & 27 \\
\hline Net lending & 18 & 9 & 9 & -18 & -44 & 7 & 7 \\
\hline Intergovernmental transfers & 79 & 63 & 89 & 137 & 280 & 387 & 481 \\
\hline Budgetary funds & 29 & 24 & 55 & 97 & 15 & 15 & 14 \\
\hline Other $1 /$ & -1 & 31 & 10 & -48 & -12 & 29 & 73 \\
\hline Interest payments & 118 & 122 & 288 & 317 & 242 & 227 & 224 \\
\hline External debt & 24 & 56 & 214 & 246 & 184 & 190 & 176 \\
\hline Domestic debt & 94 & 66 & 74 & 71 & 57 & 37 & 48 \\
\hline Primary balance (commitment) & -62 & -37 & 83 & 379 & 484 & 367 & 431 \\
\hline Overall balance (commitment) & -180 & -158 & -204 & 62 & 242 & 140 & 207 \\
\hline \multicolumn{8}{|c|}{ (In percent of GDP) } \\
\hline Revenue & 13.3 & 11.4 & 12.6 & 15.4 & 17.8 & 17.2 & 16.7 \\
\hline Cash & 10.8 & 9.2 & 12.6 & 15.4 & 17.8 & 17.2 & 16.7 \\
\hline Noncash & 2.5 & 2.1 & 0.0 & 0.0 & 0.0 & 0.0 & 0.0 \\
\hline Expenditure & 20.9 & 17.4 & 16.8 & 14.6 & 15.1 & 15.9 & 15.1 \\
\hline Interest & 5.0 & 4.6 & 6.0 & 4.3 & 2.7 & 2.1 & 1.7 \\
\hline Noninterest & 15.9 & 12.8 & 10.9 & 10.3 & 12.4 & 13.8 & 13.4 \\
\hline Primary balance (commitment) & -2.6 & -1.4 & 1.7 & 5.2 & 5.4 & 3.4 & 3.2 \\
\hline Primary balance (cash basis) & -2.2 & -0.9 & 2.1 & 4.3 & 5.4 & 3.4 & 3.2 \\
\hline Overall balance (commitment) & -7.7 & -6.0 & -4.2 & 0.8 & 2.7 & 1.3 & 1.6 \\
\hline Overall balance (cash basis) & -7.2 & -5.0 & -1.3 & 1.8 & 2.7 & 1.3 & 1.6 \\
\hline GDP (in billions of rubles) & 2,343 & 2,630 & 4,823 & 7,306 & 8,944 & 10,834 & 13,285 \\
\hline
\end{tabular}

Sources: Ministry of Finance; and Fund staff estimates.

1/ Individual expenditure categories are shown on a cash basis. Domestic expenditure arrears accumulation is included in "other" expenditure. 
Table 16. Russian Federation: Regional and Local Government Operations, 1997-2003 (In billions of rubles, unless otherwise indicated)

\begin{tabular}{|c|c|c|c|c|c|c|c|}
\hline & 1997 & 1998 & 1999 & 2000 & 2001 & 2002 & 2003 \\
\hline Revenue & 467 & 436 & 719 & 1,129 & 1,342 & 1,644 & 1,929 \\
\hline Profit tax & 69 & 61 & 139 & 221 & 300 & 291 & 356 \\
\hline Personal income tax & 73 & 71 & 97 & 147 & 253 & 358 & 456 \\
\hline VAT & 55 & 52 & 66 & 85 & 2 & 0 & 0 \\
\hline Excises & 12 & 15 & 24 & 35 & 40 & 49 & 90 \\
\hline Sales and imputed taxes & 0 & 0 & 25 & 47 & 66 & 77 & 77 \\
\hline Property tax & 47 & 47 & 52 & 63 & 88 & 119 & 136 \\
\hline Other tax revenue & 72 & 62 & 90 & 143 & 123 & 205 & 214 \\
\hline Nontax revenue & 16 & 20 & 35 & 61 & 86 & 125 & 175 \\
\hline Budgetary funds 1/ & 37 & 55 & 110 & 203 & 142 & 125 & 105 \\
\hline Transfers 2/ & 85 & 53 & 81 & 123 & 242 & 293 & 322 \\
\hline Expenditure 3/ & 489 & 469 & 721 & 1,068 & 1,364 & 1,697 & 1,999 \\
\hline Government administration & 19 & 20 & 32 & 48 & 69 & 93 & 117 \\
\hline Law enforcement & 14 & 12 & 19 & 27 & 36 & 51 & 56 \\
\hline Sectoral expenditures 4/ & 59 & 46 & 69 & 106 & 230 & 278 & 413 \\
\hline Education & 94 & 84 & 126 & 176 & 231 & 332 & 376 \\
\hline Health & 66 & 59 & 93 & 136 & 166 & 229 & 258 \\
\hline Housing & 106 & 94 & 125 & 199 & 233 & 256 & 255 \\
\hline Social security & 32 & 28 & 43 & 60 & 104 & 141 & 180 \\
\hline Budgetary funds 1 / & 42 & 61 & 112 & 203 & 131 & 154 & 149 \\
\hline Interest payments & $\ldots$ & $\ldots$ & $\ldots$ & 19 & 13 & 14 & 20 \\
\hline Other $3 /$ & 55 & 64 & 102 & 94 & 149 & 152 & 175 \\
\hline Overall balance (- deficit) & -22 & -33 & -1 & 62 & -22 & -53 & -70 \\
\hline Financing & 22 & 33 & 1 & -62 & 22 & 53 & 70 \\
\hline Foreign & 5 & 4 & 0 & -28 & -4 & -4 & -4 \\
\hline Domestic & 17 & 15 & 2 & -34 & 19 & 55 & 62 \\
\hline Monetary authorities & -2 & 2 & -7 & -18 & 5 & -8 & -9 \\
\hline Commercial banks & 5 & 8 & -6 & -23 & 0 & 33 & 28 \\
\hline Other & 14 & 6 & 15 & 7 & 15 & 29 & 43 \\
\hline Arrears and rescheduling & 0 & 14 & -1 & 0 & 7 & 3 & 11 \\
\hline \multirow[t]{2}{*}{ Domestic expenditure 4/ } & 0 & 14 & -1 & 0 & 7 & 3 & 11 \\
\hline & \multicolumn{6}{|c|}{ (In percent of GDP) } & \\
\hline Revenue & 19.9 & 16.6 & 14.9 & 15.5 & 15.0 & 15.2 & 14.5 \\
\hline Profit tax & 2.9 & 2.3 & 2.9 & 3.0 & 3.4 & 2.7 & 2.7 \\
\hline Personal income tax & 3.1 & 2.7 & 2.0 & 2.0 & 2.8 & 3.3 & 3.4 \\
\hline VAT & 2.3 & 2.0 & 1.4 & 1.2 & 0.0 & 0.0 & 0.0 \\
\hline Excises & 0.5 & 0.6 & 0.5 & 0.5 & 0.4 & 0.5 & 0.7 \\
\hline Sales and imputed taxes & 0.0 & 0.0 & 0.5 & 0.6 & 0.7 & 0.7 & 0.6 \\
\hline Property tax & 2.0 & 1.8 & 1.1 & 0.9 & 1.0 & 1.1 & 1.0 \\
\hline Other tax revenue & 3.1 & 2.4 & 1.9 & 2.0 & 1.4 & 1.9 & 1.6 \\
\hline Nontax revenue & 0.7 & 0.8 & 0.7 & 0.8 & 1.0 & 1.2 & 1.3 \\
\hline Budgetary funds $1 /$ & 1.6 & 2.1 & 2.3 & 2.8 & 1.6 & 1.2 & 0.8 \\
\hline Transfers 2/ & 3.6 & 2.0 & 1.7 & 1.7 & 2.7 & 2.7 & 2.4 \\
\hline Expenditure 3/ & 20.9 & 17.8 & 14.9 & 14.6 & 15.2 & 15.7 & 15.0 \\
\hline Government administration & 0.8 & 0.8 & 0.7 & 0.7 & 0.8 & 0.9 & 1.1 \\
\hline Law enforcement & 0.6 & 0.5 & 0.4 & 0.4 & 0.4 & 0.5 & 0.5 \\
\hline Sectoral expenditures 4/ & 2.5 & 1.8 & 1.4 & 1.4 & 2.6 & 2.6 & 3.8 \\
\hline Education & 4.0 & 3.2 & 2.6 & 2.4 & 2.6 & 3.1 & 3.5 \\
\hline Health & 2.8 & 2.2 & 1.9 & 1.9 & 1.9 & 2.1 & 2.4 \\
\hline Housing & 4.5 & 3.6 & 2.6 & 2.7 & 2.6 & 2.4 & 2.4 \\
\hline Social security & 1.4 & 1.1 & 0.9 & 0.8 & 1.2 & 1.3 & 1.7 \\
\hline Budgetary funds $1 /$ & 1.8 & 2.3 & 2.3 & 2.8 & 1.5 & 1.4 & 1.4 \\
\hline Interest payments & $\ldots$ & $\ldots$ & $\ldots$ & 0.3 & 0.1 & 0.1 & 0.2 \\
\hline Other $3 /$ & 0.8 & 0.9 & 1.4 & 1.3 & 1.7 & 1.4 & 1.6 \\
\hline Overall balance (- deficit) & -0.9 & -1.2 & 0.0 & 0.8 & -0.2 & -0.5 & -0.5 \\
\hline Financing & 0.9 & 1.2 & 0.0 & -0.8 & 0.2 & 0.5 & 0.5 \\
\hline Foreign & 0.2 & 0.2 & 0.0 & -0.4 & 0.0 & 0.0 & 0.0 \\
\hline Domestic & 0.7 & 0.6 & 0.0 & -0.5 & 0.2 & 0.5 & 0.6 \\
\hline Monetary authorities & -0.1 & 0.1 & -0.1 & -0.2 & 0.1 & -0.1 & -0.1 \\
\hline Commercial banks & 0.2 & 0.3 & -0.1 & -0.3 & 0.0 & 0.3 & 0.3 \\
\hline Other & 0.6 & 0.2 & 0.3 & 0.1 & 0.2 & 0.3 & 0.4 \\
\hline Arrears and rescheduling & 0.0 & 0.5 & 0.0 & 0.0 & 0.1 & 0.0 & 0.1 \\
\hline Domestic expenditure 4/ & 0.0 & 0.5 & 0.0 & 0.0 & 0.1 & 0.0 & 0.1 \\
\hline
\end{tabular}

Sources: Ministry of Finance, CBR and Fund staff estimates.

$1 /$ Including all territorial road funds.

2/ Including net budgetary loans.

3/ Individual expenditure categories shown on a cash basis. Domestic expenditure arrears accumulation is included in "other" expenditure.

4/ Including research; industry, energy and construction; agriculture and fishing; transport and communications; environment. 
Table 17. Russian Federation: Extrabudgetary Fund Operations, 1997-2003 (In billions of rubles, unless otherwise indicated)

\begin{tabular}{|c|c|c|c|c|c|c|c|}
\hline & 1997 & 1998 & 1999 & 2000 & 2001 & 2002 & 2003 \\
\hline Revenue & 251 & 234 & 391 & 604 & 713 & 956 & 1,134 \\
\hline Pension Fund & 181 & 162 & 277 & 424 & 531 & 706 & 827 \\
\hline Employment Fund & 9 & 8 & 13 & 20 & 0 & 0 & 0 \\
\hline Social Insurance Fund & 32 & 33 & 52 & 87 & 89 & 116 & 143 \\
\hline Medical Insurance Fund & 30 & 31 & 49 & 73 & 93 & 133 & 164 \\
\hline Expenditure & 248 & 258 & 335 & 498 & 691 & 979 & 1,126 \\
\hline Pension Fund 1/ & 177 & 190 & 235 & 341 & 515 & 721 & 815 \\
\hline Employment Fund & 9 & 8 & 10 & 16 & 0 & 0 & 0 \\
\hline Social Insurance Fund & 30 & 32 & 43 & 70 & 93 & 126 & 149 \\
\hline Medical Insurance Fund & 29 & 32 & 47 & 71 & 91 & 135 & 160 \\
\hline Float & 3 & -3 & 0 & 1 & -9 & -2 & 2 \\
\hline Balance, total extrabudgetary funds & 3 & -24 & 56 & 106 & 22 & -23 & 8 \\
\hline Financing & -3 & 24 & -56 & -106 & -22 & 23 & -8 \\
\hline of which: monetary authorities & -2 & -1 & -10 & -107 & -12 & 97 & -25 \\
\hline of which: pension arrears & $\cdots$ & 26 & -29 & -2 & 0 & 0 & 0 \\
\hline & \multicolumn{7}{|c|}{ (In percent of GDP) } \\
\hline Revenue & 10.7 & 8.9 & 8.1 & 8.3 & 8.0 & 8.8 & 8.5 \\
\hline Pension Fund & 7.7 & 6.1 & 5.7 & 5.8 & 5.9 & 6.5 & 6.2 \\
\hline Employment Fund & 0.4 & 0.3 & 0.3 & 0.3 & 0.0 & 0.0 & 0.0 \\
\hline Social Insurance Fund & 1.3 & 1.2 & 1.1 & 1.2 & 1.0 & 1.1 & 1.1 \\
\hline Medical Insurance Fund & 1.3 & 1.2 & 1.0 & 1.0 & 1.0 & 1.2 & 1.2 \\
\hline Expenditure & 10.6 & 9.8 & 6.9 & 6.8 & 7.7 & 9.0 & 8.5 \\
\hline Pension Fund 1/ & 7.5 & 7.2 & 4.9 & 4.7 & 5.8 & 6.7 & 6.1 \\
\hline Employment Fund & 0.4 & 0.3 & 0.2 & 0.2 & 0.0 & 0.0 & 0.0 \\
\hline Social Insurance Fund & 1.3 & 1.2 & 0.9 & 1.0 & 1.0 & 1.2 & 1.1 \\
\hline Medical Insurance Fund & 1.2 & 1.2 & 1.0 & 1.0 & 1.0 & 1.2 & 1.2 \\
\hline Float & 0.1 & -0.1 & 0.0 & 0.0 & -0.1 & 0.0 & 0.0 \\
\hline Balance, total extrabudgetary funds & 0.1 & -0.9 & 1.2 & 1.4 & 0.2 & -0.2 & 0.1 \\
\hline Financing & -0.1 & 0.9 & -1.2 & -1.4 & -0.2 & 0.2 & -0.1 \\
\hline of which: monetary authorities & -0.1 & 0.0 & -0.2 & -1.5 & -0.1 & 0.9 & -0.2 \\
\hline of which: pension arrears & $\ldots$ & 1.0 & -0.6 & 0.0 & 0.0 & 0.0 & 0.0 \\
\hline
\end{tabular}

Sources: Extrabudgetary funds; and CBR.

1/ Measured on a cash basis prior to 1998 . 


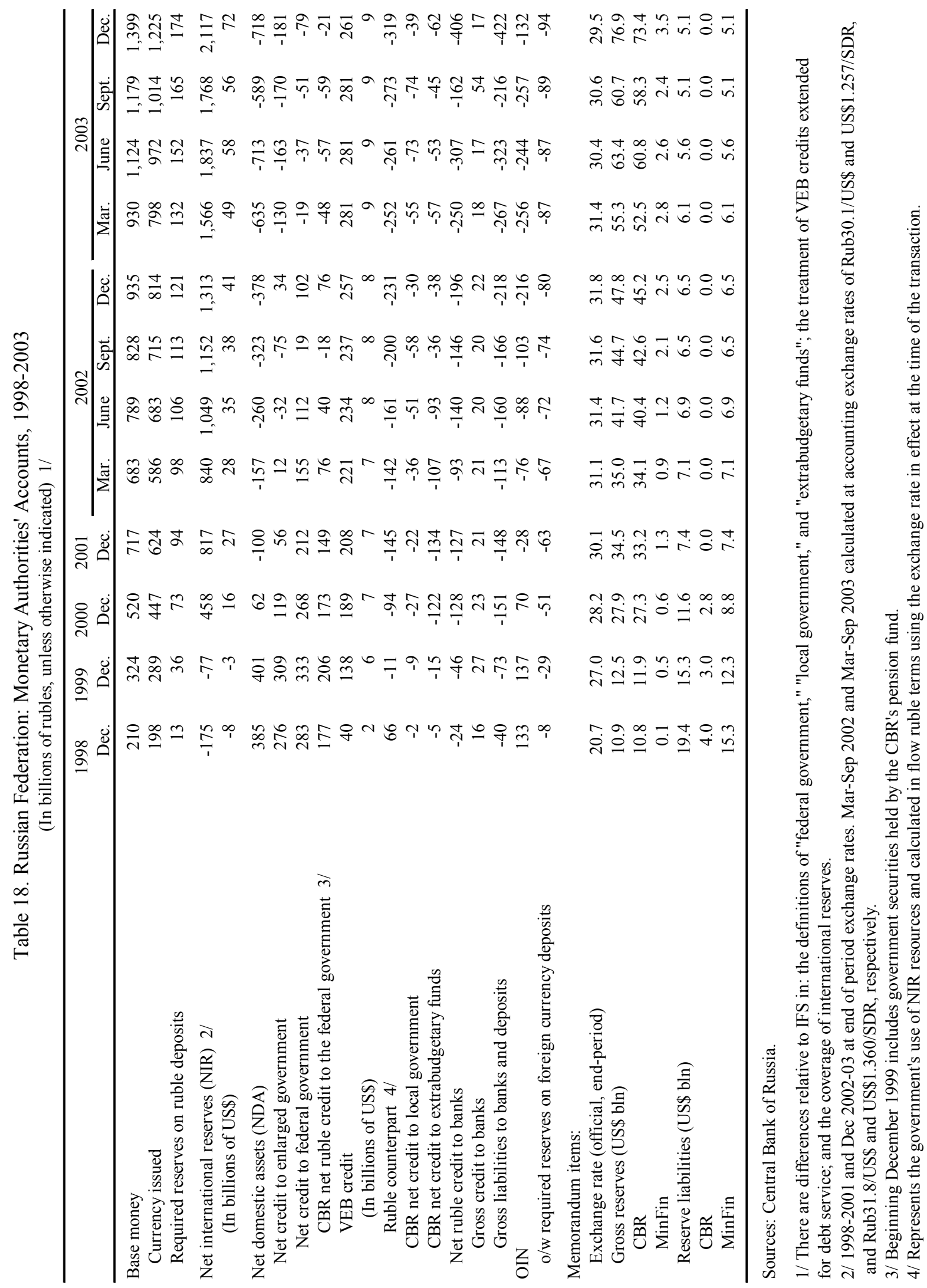




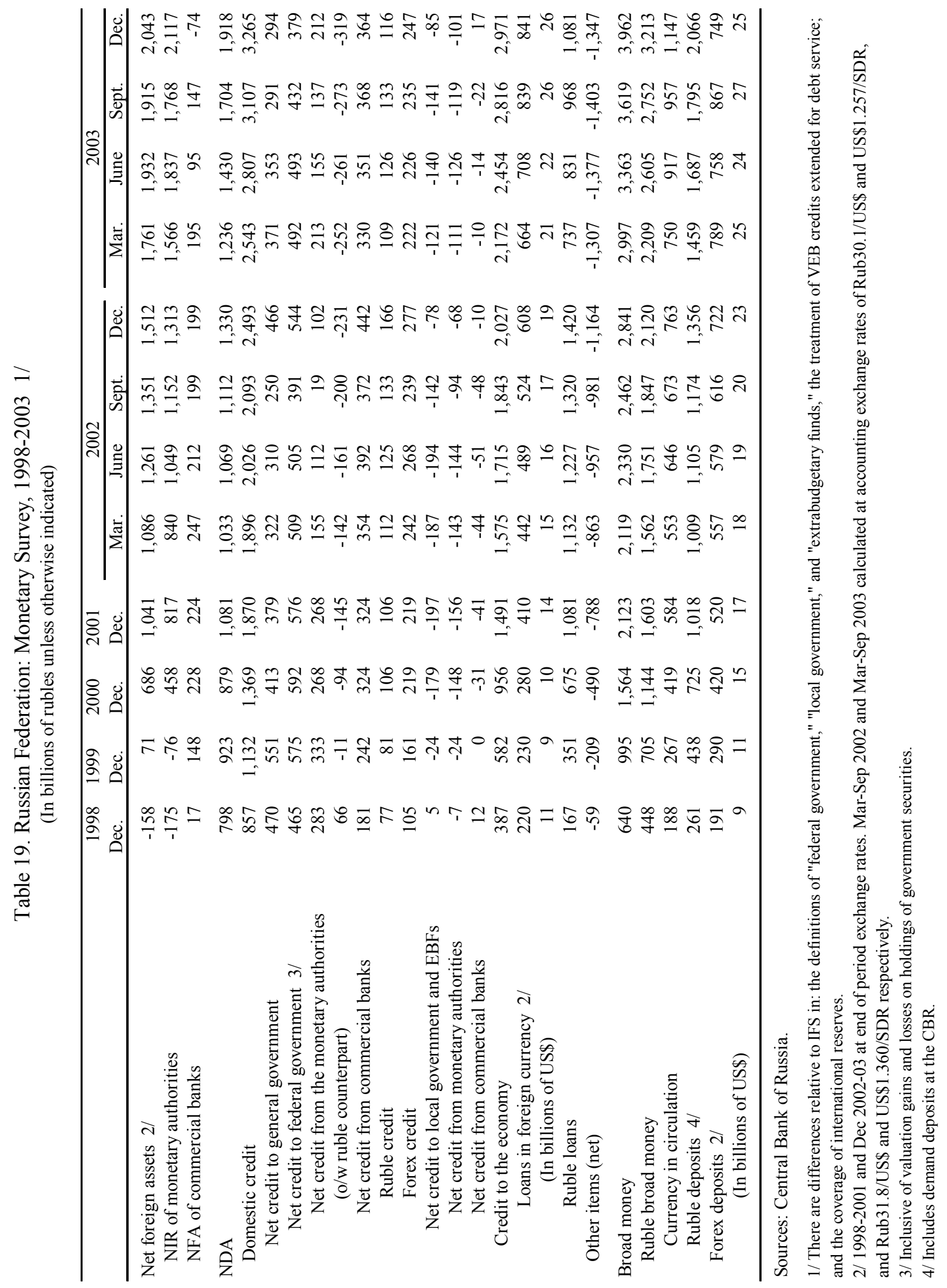


Table 20. Russian Federation: Key Monetary Indicators, 1998-2003

\begin{tabular}{|c|c|c|c|c|c|c|c|c|c|c|c|c|}
\hline & \multirow{2}{*}{$\begin{array}{c}1998 \\
\text { Dec. }\end{array}$} & \multirow{2}{*}{$\begin{array}{r}1999 \\
\text { Dec. }\end{array}$} & \multirow{2}{*}{$\begin{array}{r}2000 \\
\text { Dec. }\end{array}$} & \multirow{2}{*}{$\begin{array}{c}2001 \\
\text { Dec. }\end{array}$} & \multicolumn{4}{|c|}{2002} & \multicolumn{4}{|c|}{2003} \\
\hline & & & & & Mar. & June & Sep. & $\overline{\text { Dec. }}$ & Mar. & June & Sep. & Dec. \\
\hline \multicolumn{13}{|l|}{ Velocity } \\
\hline Ruble broad money velocity 1 / & 7.3 & 8.1 & 6.8 & 6.1 & 5.8 & 5.8 & 6.5 & 5.7 & 5.2 & 4.8 & 5.2 & 4.6 \\
\hline$\%$ change compared to year earlier & -1.5 & 10.8 & -15.4 & -10.5 & -12.2 & -11.2 & -7.0 & -6.9 & -9.8 & -17.2 & -20.0 & -19.6 \\
\hline Ruble broad money velocity (seasonally adj.) 1/ & 7.3 & 8.1 & 6.8 & 6.1 & 6.1 & 6.0 & 6.0 & 5.7 & 5.5 & 5.0 & 4.8 & 4.6 \\
\hline$\%$ change compared to year earlier & -1.5 & 10.8 & -15.4 & -10.5 & -12.2 & -11.2 & -7.0 & -6.9 & -9.8 & -17.2 & -20.0 & -19.6 \\
\hline Broad money including forex deposits $1 /$ & 5.1 & 5.9 & 5.1 & 4.6 & 4.3 & 4.3 & 4.9 & 4.2 & 3.9 & 3.7 & 4.0 & 3.7 \\
\hline$\%$ change compared to year earlier & -14.8 & 15.1 & -14.4 & -8.7 & -8.4 & -8.1 & -6.1 & -9.5 & -9.9 & -14.7 & -18.8 & -12.5 \\
\hline Ruble money multiplier & 2.15 & 2.11 & 2.20 & 2.23 & 2.29 & 2.22 & 2.23 & 2.27 & 2.37 & 2.32 & 2.33 & 2.30 \\
\hline Currency-to-deposit ratio & 0.71 & 0.61 & 0.58 & 0.57 & 0.55 & 0.58 & 0.57 & 0.56 & 0.51 & 0.54 & 0.53 & 0.56 \\
\hline Reserves -to-deposits ratio & 0.05 & 0.08 & 0.10 & 0.09 & 0.10 & 0.10 & 0.10 & 0.09 & 0.09 & 0.09 & 0.09 & 0.08 \\
\hline Currency held by banks to deposits ratio & 0.04 & 0.05 & 0.04 & 0.04 & 0.03 & 0.03 & 0.04 & 0.04 & 0.03 & 0.03 & 0.03 & 0.04 \\
\hline \multicolumn{13}{|l|}{ Currency ratios } \\
\hline Forex deposits-to-total deposits ratio & 0.42 & 0.40 & 0.36 & 0.34 & 0.36 & 0.34 & 0.34 & 0.35 & 0.35 & 0.31 & 0.33 & 0.27 \\
\hline Forex credit-to-total credit ratio & 0.57 & 0.40 & 0.29 & 0.28 & 0.28 & 0.28 & 0.28 & 0.30 & 0.31 & 0.29 & 0.30 & 0.28 \\
\hline \multicolumn{13}{|l|}{ Real measures of monetary aggregates } \\
\hline Real currency in circulation $2 /$ & 0.91 & 0.95 & 1.24 & 1.45 & 1.31 & 1.47 & 1.52 & 1.65 & 1.54 & 1.84 & 1.91 & 2.22 \\
\hline Real ruble deposits $2 /$ & 0.75 & 0.91 & 1.25 & 1.48 & 1.39 & 1.47 & 1.54 & 1.71 & 1.75 & 1.97 & 2.08 & 2.32 \\
\hline Real ruble broad money $2 /$ & 0.81 & 0.92 & 1.25 & 1.47 & 1.36 & 1.47 & 1.53 & 1.69 & 1.67 & 1.92 & 2.02 & 2.28 \\
\hline Real forex deposits $2 /$ & 0.89 & 0.88 & 1.14 & 1.24 & 1.30 & 1.32 & 1.40 & 1.49 & 1.53 & 1.39 & 1.59 & 1.38 \\
\hline Real credit (incl. Forex ) to the economy 2/ & 0.55 & 0.60 & 0.82 & 1.08 & 1.09 & 1.14 & 1.21 & 1.28 & 1.30 & 1.44 & 1.64 & 1.68 \\
\hline Real ruble credit to the economy 2 / & 0.35 & 0.53 & 0.84 & 1.13 & 1.12 & 1.18 & 1.25 & 1.29 & 1.30 & 1.47 & 1.65 & 1.73 \\
\hline \multicolumn{13}{|l|}{ Contributions to monetary growth } \\
\hline \multicolumn{13}{|l|}{ 1. Monetary authorities $3 /$} \\
\hline Base money growth & 27.9 & 54.1 & 60.2 & 38.1 & -4.8 & 10.0 & 15.5 & 30.4 & -0.5 & 20.2 & 26.1 & 49.6 \\
\hline NDA & 65.2 & -7.9 & -95.5 & -31.1 & -7.9 & -22.4 & -31.1 & -38.7 & -27.5 & -35.9 & -22.6 & -36.4 \\
\hline Net credit to enlarged government & 51.7 & 13.7 & -72.1 & -12.2 & -6.2 & -12.2 & -18.2 & -3.0 & -17.6 & -21.1 & -21.9 & -23.0 \\
\hline NIR & -37.3 & 62.0 & 155.8 & 69.1 & 3.1 & 32.3 & 46.6 & 69.1 & 27.0 & 56.0 & 48.7 & 86.0 \\
\hline \multicolumn{13}{|l|}{ 2. Banking system 4/ } \\
\hline Broad money & 41.3 & 43.6 & 55.5 & 37.2 & -0.2 & 9.8 & 16.0 & 33.9 & 5.5 & 18.3 & 27.4 & 39.4 \\
\hline NDA & 42.1 & 7.3 & -2.3 & 11.7 & -2.3 & -0.6 & 1.4 & 11.7 & -3.3 & 3.5 & 13.2 & 20.7 \\
\hline Net credit to enlarged government & 32.4 & 6.3 & -19.1 & 1.2 & -2.7 & -3.2 & -6.1 & 4.1 & -3.3 & -4.0 & -6.1 & -6.0 \\
\hline NFA & -0.8 & 36.3 & 57.8 & 25.5 & 2.1 & 10.4 & 14.6 & 22.2 & 8.8 & 14.8 & 14.2 & 18.7 \\
\hline \multicolumn{13}{|l|}{ Credit aggregates--stocks 5/ } \\
\hline Total credit to the economy & 14.7 & 12.2 & 13.4 & 16.7 & 14.5 & 15.8 & 17.0 & 18.7 & 16.4 & 18.5 & 21.2 & 22.4 \\
\hline Ruble-denominated credit to the economy & 6.4 & 7.4 & 9.6 & 12.1 & 10.5 & 11.3 & 12.2 & 13.1 & 11.3 & 13.1 & 14.9 & 16.0 \\
\hline Credit to government from commercial banks & 8.8 & 4.7 & 4.0 & 3.6 & 2.9 & 3.2 & 3.0 & 4.0 & 3.8 & 3.9 & 3.5 & 3.6 \\
\hline \multicolumn{13}{|l|}{ Credit aggregates--flows 5/ } \\
\hline Total credit to the economy & 3.6 & 4.1 & 5.5 & 6.0 & 0.8 & 2.1 & 3.3 & 5.0 & 1.1 & 3.2 & 5.9 & 7.1 \\
\hline Ruble-denominated credit to the economy & -0.7 & 3.8 & 4.6 & 4.5 & 0.5 & 1.3 & 2.2 & 3.1 & 0.7 & 2.5 & 4.2 & 5.3 \\
\hline Credit to government from commercial banks & 2.3 & 0.6 & 0.9 & 0.3 & -0.1 & 0.2 & 0.0 & 1.0 & 0.5 & 0.6 & 0.2 & 0.3 \\
\hline \multicolumn{13}{|l|}{ Prices } \\
\hline Inflation from end of previous year & 84.4 & 36.5 & 20.2 & 18.6 & 5.5 & 9.1 & 10.4 & 15.1 & 14.8 & 13.9 & 13.2 & 12.0 \\
\hline \multicolumn{13}{|c|}{ Source: Central Bank of Russia and Fund staff estimates. } \\
\hline \multicolumn{13}{|c|}{$\begin{array}{l}\text { 1/ Based on annualized end-period quarterly GDP. } \\
\text { 2/ End-1994 = 1.00. Deflated by the CPI, stocks of foreign currency-denominated items converted into rubles at prevailing exchange rates. } \\
\text { 3/ Change as a percent of beginning of year stock of base money. Changes in NIR include valuation effects arising from exchange rate movements. } \\
\text { 4/ Change as a percent of beginning of year broad money (including foreign exchange deposits). Changes in NFA include valuation changes arising } \\
\text { from exchange rate movements. } \\
\text { 5/ Relative to annual GDP. }\end{array}$} \\
\hline
\end{tabular}


웅유

Ð

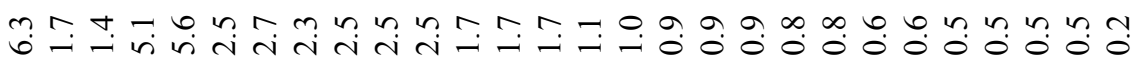

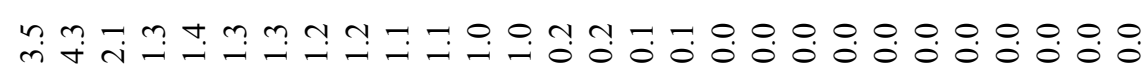

ஹீ요

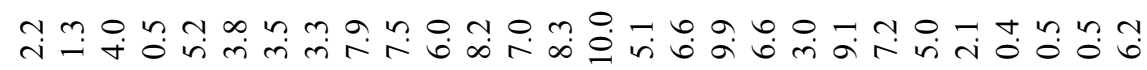

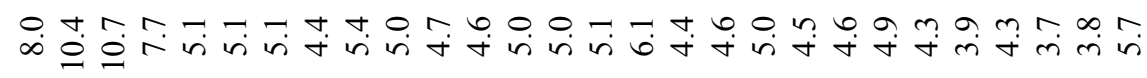

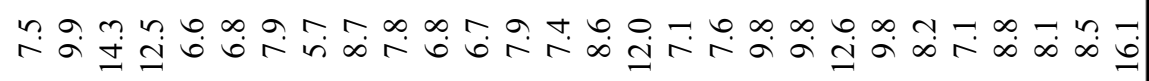

nm

ஸึ่

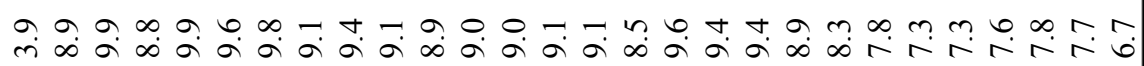

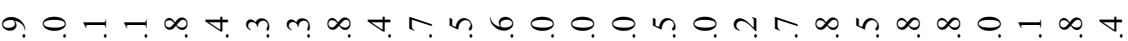

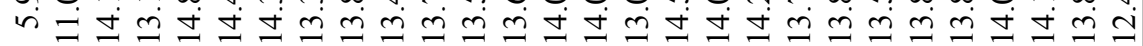

बे

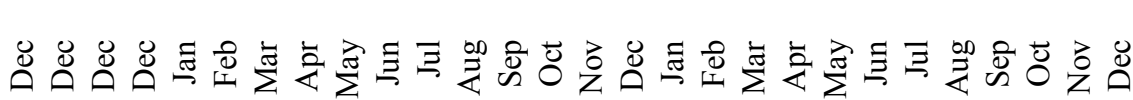

ஃ。 
Table 22. Russian Federation: Domestic Debt, 1998-2003 1/

(In billions of rubles)

\begin{tabular}{|c|c|c|c|c|c|c|}
\hline & 1998 & 1999 & 2000 & 2001 & 2002 & 2003 \\
\hline Short-term treasury bills (GKO) & 17 & 7 & 3 & 20 & 19 & 3 \\
\hline Medium and long-term government bonds (OFZ's) & 460 & 515 & 506 & 470 & 624 & 649 \\
\hline OFZ-PD (fixed coupon) & 347 & 402 & 359 & 308 & 351 & 50 \\
\hline OFZ-FK (fixed coupon) & 113 & 112 & 123 & 139 & 207 & 199 \\
\hline OFZ-PK (variable coupon) & 0 & 0 & 24 & 24 & 24 & 24 \\
\hline OFZ-AD & 0 & 0 & 0 & 0 & 42 & 375 \\
\hline Nonmarket bonds (OGNZ) & 3 & 3 & 20 & 21 & 12 & 12 \\
\hline Savings bonds (OGSZ) & 15 & 5 & 3 & 0 & 0 & 0 \\
\hline Short-term bank loans & 0 & 15 & 0 & 0 & 0 & 0 \\
\hline Other $2 /$ & 36 & 34 & 26 & 23 & 26 & 18 \\
\hline Total & 530 & 578 & 557 & 533 & 680 & 682 \\
\hline
\end{tabular}

Source: Ministry of Finance.

1/ Ruble denominated debt. Includes instruments held by nonresidents. Excludes obligations of the ministry of finance to the CBR as well as eurobonds held by residents.

2/ Includes targeted bond issues, various government guarantees, and enterprise/sector debts assumed by the government. 
Table 23. Russian Federation: Balance of Payments, 1997-2003 (In billions of U.S. dollars, unless otherwise indicated)

\begin{tabular}{|c|c|c|c|c|c|c|c|}
\hline & 1997 & 1998 & 1999 & 2000 & 2001 & 2002 & 2003 \\
\hline Current Account & -2.6 & -2.1 & 22.2 & 44.6 & 33.4 & 30.9 & 35.8 \\
\hline Trade Balance & 14.9 & 16.4 & 36.0 & 60.2 & 48.1 & 46.3 & 60.5 \\
\hline Exports & 86.9 & 74.4 & 75.6 & 105.0 & 101.9 & 107.2 & 135.9 \\
\hline Non-energy & 48.4 & 46.5 & 44.6 & 52.2 & 50.2 & 51.8 & 62.2 \\
\hline Energy & 38.5 & 27.9 & 31.0 & 52.8 & 51.7 & 55.5 & 73.7 \\
\hline Oil & 22.1 & 14.5 & 19.6 & 36.2 & 34.0 & 39.6 & 53.7 \\
\hline Gas & 16.4 & 13.4 & 11.4 & 16.6 & 17.8 & 15.9 & 20.0 \\
\hline Imports & -72.0 & -58.0 & -39.5 & -44.9 & -53.8 & -61.0 & -75.4 \\
\hline Services (net) & -17.1 & -18.2 & -14.4 & -15.6 & -13.9 & -15.0 & -24.3 \\
\hline Nonfactor services & -5.9 & -4.1 & -4.3 & -6.7 & -8.4 & -9.1 & -11.1 \\
\hline Factor services & -11.2 & -14.1 & -10.1 & -8.9 & -5.5 & -6.0 & -13.2 \\
\hline Public sector interest & -10.0 & -12.3 & -8.9 & -8.4 & -6.6 & -5.6 & -5.2 \\
\hline Other factor services & -1.2 & -1.8 & -1.2 & -0.5 & 1.1 & -0.3 & -7.9 \\
\hline Current transfers & -0.4 & -0.3 & 0.6 & 0.1 & -0.8 & -0.3 & -0.4 \\
\hline Capital and financial and account & 33.1 & -5.2 & -14.6 & -21.8 & -13.9 & -10.9 & -0.6 \\
\hline Capital transfers & -0.8 & -0.4 & -0.3 & -0.3 & -0.5 & -0.5 & -1.0 \\
\hline Federal capital & 42.2 & 7.2 & -1.4 & -4.3 & -6.8 & -6.6 & -4.4 \\
\hline Budgetary & 1.3 & 0.4 & -5.4 & -5.4 & -5.2 & -4.9 & -7.4 \\
\hline Disbursements & 9.0 & 8.7 & 2.1 & 1.1 & 0.6 & 0.7 & 0.8 \\
\hline Amortization & -7.7 & -8.3 & -7.5 & -6.6 & -5.8 & -5.5 & -8.2 \\
\hline Non-budgetary & 41.0 & 6.8 & 4.0 & 1.1 & -1.6 & -1.8 & 3.0 \\
\hline Local governments & 1.2 & 0.8 & -0.3 & -0.8 & -0.2 & -0.5 & -0.2 \\
\hline Private sector capital & -9.5 & -12.8 & -12.6 & -16.3 & -6.4 & -3.3 & 5.0 \\
\hline Direct investment & 1.6 & 1.2 & 0.6 & -0.4 & -0.2 & -1.2 & -3.0 \\
\hline Portfolio investment (net) & 1.3 & 0.8 & -0.2 & -0.2 & 0.6 & 2.0 & -0.8 \\
\hline Commercial banks & 7.6 & -6.0 & -4.3 & -2.1 & 1.0 & 2.2 & 10.3 \\
\hline Corporations & 6.2 & 4.6 & -0.6 & -1.0 & 0.4 & 8.3 & 12.0 \\
\hline Other private capital & -26.2 & -13.4 & -8.1 & -12.7 & -8.2 & -14.7 & -13.4 \\
\hline Errors and omissions, net & -8.8 & -9.8 & -8.6 & -9.2 & -10.2 & -7.5 & -5.2 \\
\hline Overall balance & 21.8 & -17.1 & -0.9 & 13.7 & 9.3 & 12.5 & 30.1 \\
\hline Financing & -21.8 & 17.1 & 0.9 & -13.7 & -9.3 & -12.5 & -30.1 \\
\hline Net international reserves & -2.0 & 10.9 & -5.4 & -18.7 & -10.7 & -13.0 & -31.2 \\
\hline Gross reserves ( - increase) & -2.5 & 5.6 & -1.7 & -15.8 & -8.8 & -9.4 & -29.1 \\
\hline Net Fund liabilities & 1.5 & 5.3 & -3.6 & -2.9 & -3.8 & -1.5 & -2.0 \\
\hline Purchases & 2.0 & 6.2 & 0.6 & 0.0 & 0.0 & 0.0 & 0.0 \\
\hline Repurchases & -0.5 & -0.9 & -4.2 & -2.9 & -3.8 & -1.5 & -2.0 \\
\hline Other liabilities & -1.1 & 0.1 & -0.1 & -0.1 & 1.9 & -2.1 & 0.0 \\
\hline Valuation adjustment & 1.6 & -0.4 & 0.1 & -0.2 & 0.7 & -0.8 & 3.2 \\
\hline Exceptional financing 1/ & -21.4 & 6.6 & 6.3 & 5.2 & 0.7 & 1.3 & -2.1 \\
\hline Change in arrears & -24.5 & 4.3 & 1.7 & 1.4 & 0.7 & -0.4 & -2.6 \\
\hline Reschedulings & 3.1 & 2.2 & 4.5 & 3.8 & 0.1 & 1.7 & 0.5 \\
\hline \multicolumn{8}{|l|}{ Memorandum items: } \\
\hline Current account (in percent of GDP) & -0.6 & -0.7 & 11.6 & 17.2 & 10.9 & 9.0 & 8.3 \\
\hline Gross reserves $2 /$ & 17.8 & 10.9 & 12.4 & 27.9 & 34.5 & 47.8 & 76.9 \\
\hline (in months of imports of GNFS) & 2.9 & 2.5 & 2.4 & 4.6 & 5.0 & 5.6 & 7.1 \\
\hline (percent of short-term debt by remaining maturity) 3 / & $\ldots$ & $\ldots$ & 52 & 89 & 147 & 156 & 235 \\
\hline (percent of public debt service) & 90 & 66 & 83 & 132 & 309 & 315 & 684 \\
\hline Net private capital outflows & -18.3 & -22.6 & -21.2 & -25.5 & -16.7 & -10.8 & -0.1 \\
\hline Net private capital outflows (in percent of trade) & -11.5 & -17.0 & 18.4 & 17.0 & 10.7 & 6.4 & 0.1 \\
\hline World oil price (\$barrel) & 19.2 & 13.1 & 18.0 & 28.2 & 24.3 & 25.0 & 28.9 \\
\hline Public external debt service payments 4 / & 15.1 & 19.9 & 16.6 & 14.9 & 17.0 & 11.2 & 15.2 \\
\hline (percent of exports of goods and services) & 15.0 & 22.9 & 19.6 & 13.0 & 15.1 & 9.3 & 10.0 \\
\hline
\end{tabular}

Sources: Russian authorities; and Fund staff estimates. Data are presented on a due basis.

1/ Includes arrears, debt rescheduling, and debt deferrals.

2/ Excluding repos with non-residents to avoid double counting of reserve assets.

3/ Excludes arrears.

4/ Net of rescheduling. 
Table 24. Russian Federation: Destination of Exports, 1997 - 2003 1/

\begin{tabular}{|c|c|c|c|c|c|c|c|}
\hline & 1997 & 1998 & 1999 & 2000 & 2001 & 2002 & 2003 \\
\hline & \multicolumn{7}{|c|}{ (In millions of US dollars) } \\
\hline Total exports & 85,036 & 71,313 & 72,885 & 103,093 & 99,970 & 106,712 & 133,450 \\
\hline CIS & 16,624 & 13,699 & 10,707 & 13,824 & 14,617 & 15,711 & 20,450 \\
\hline Belarus & 4,673 & 4,670 & 3,767 & 5,572 & 5,348 & 5,922 & 7,559 \\
\hline Kazakhstan & 2,471 & 1,893 & 1,222 & 2,246 & 2,778 & 2,403 & 3,279 \\
\hline Ukraine & 7,239 & 5,560 & 4,792 & 5,024 & 5,282 & 5,885 & 7,595 \\
\hline Other & 2,240 & 1,576 & 926 & 982 & 1,209 & 1,500 & 2,017 \\
\hline Non-CIS & 68,412 & 57,615 & 62,179 & 89,269 & 85,353 & 91,001 & 113,000 \\
\hline Europe & 46,442 & 37,810 & 40,490 & 59,660 & 56,092 & 59,662 & 72,462 \\
\hline Czech Republic & 1,823 & 1,396 & 1,323 & 1,745 & 1,669 & 1,511 & 1,941 \\
\hline Finland & 2,774 & 2,071 & 2,414 & 3,105 & 3,113 & 2,935 & 4,319 \\
\hline France & 1,626 & 1,456 & 1,211 & 1,903 & 2,250 & 2,659 & 3,491 \\
\hline Germany & 6,531 & 5,719 & 6,205 & 9,231 & 9,194 & 8,060 & 10,419 \\
\hline Hungary & 1,854 & 1,487 & 1,547 & 2,405 & 2,379 & 2,167 & 2,821 \\
\hline Ireland & 2,500 & 643 & 658 & 288 & 117 & 260 & 924 \\
\hline Italy & 3,564 & 3,219 & 3,755 & 7,254 & 7,401 & 7,441 & 8,512 \\
\hline Netherlands & 4,554 & 3,959 & 3,673 & 4,349 & 4,695 & 7,529 & 8,664 \\
\hline Poland & 2,514 & 2,180 & 2,606 & 4,452 & 4,200 & 3,720 & 4,619 \\
\hline Slovak Republic & 1,740 & 1,372 & 1,426 & 2,121 & 2,205 & 2,032 & 2,297 \\
\hline Switzerland & 3,542 & 3,118 & 3,351 & 3,857 & 2,309 & 5,360 & 5,807 \\
\hline UK & 2,846 & 2,960 & 2,886 & 4,670 & 4,217 & 3,803 & 4,873 \\
\hline Other & 10,576 & 8,230 & 9,435 & 14,280 & 12,342 & 12,185 & 13,774 \\
\hline Asia & 12,891 & 9,556 & 10,972 & 16,948 & 17,035 & 19,078 & 27,199 \\
\hline China & 3,982 & 3,169 & 3,527 & 5,248 & 5,596 & 6,837 & 8,252 \\
\hline Japan & 2,935 & 2,176 & 2,125 & 2,766 & 2,427 & 1,803 & 2,420 \\
\hline Other & 5,974 & 4,211 & 5,320 & 8,934 & 9,012 & 10,438 & 16,527 \\
\hline Western Hemisphere & 6,719 & 7,350 & 7,705 & 9,158 & 8,207 & 7,454 & 8,024 \\
\hline US & 4,482 & 5,100 & 4,709 & 4,644 & 4,198 & 3,989 & 4,206 \\
\hline Other & 2,237 & 2,250 & 2,996 & 4,514 & 4,009 & 3,466 & 3,818 \\
\hline Middle East and Africa & 2,124 & 2,789 & 2,770 & 3,459 & 3,966 & 4,717 & 5,220 \\
\hline Other & 236 & 110 & 242 & 44 & 53 & 90 & 95 \\
\hline Exports to: & \multicolumn{7}{|c|}{ (In percent of total exports) } \\
\hline CIS & 19.5 & 19.2 & 14.7 & 13.4 & 14.6 & 14.7 & 15.3 \\
\hline Belarus & 5.5 & 6.5 & 5.2 & 5.4 & 5.3 & 5.5 & 5.7 \\
\hline Kazakhstan & 2.9 & 2.7 & 1.7 & 2.2 & 2.8 & 2.3 & 2.5 \\
\hline Ukraine & 8.5 & 7.8 & 6.6 & 4.9 & 5.3 & 5.5 & 5.7 \\
\hline Other & 2.6 & 2.2 & 1.3 & 1.0 & 1.2 & 1.4 & 1.5 \\
\hline Non-CIS & 80.5 & 80.8 & 85.3 & 86.6 & 85.4 & 85.3 & 84.7 \\
\hline Europe & 54.6 & 53.0 & 55.6 & 57.9 & 56.1 & 55.8 & 54.3 \\
\hline Czech Republic & 2.1 & 2.0 & 1.8 & 1.7 & 1.7 & 1.4 & 1.5 \\
\hline Finland & 3.3 & 2.9 & 3.3 & 3.0 & 3.1 & 2.8 & 3.2 \\
\hline France & 1.9 & 2.0 & 1.7 & 1.8 & 2.3 & 2.5 & 2.6 \\
\hline Germany & 7.7 & 8.0 & 8.5 & 9.0 & 9.2 & 7.6 & 7.8 \\
\hline Hungary & 2.2 & 2.1 & 2.1 & 2.3 & 2.4 & 2.0 & 2.1 \\
\hline Ireland & 2.9 & 0.9 & 0.9 & 0.3 & 0.1 & 0.2 & 0.7 \\
\hline Italy & 4.2 & 4.5 & 5.2 & 7.0 & 7.4 & 7.0 & 6.4 \\
\hline Netherlands & 5.4 & 5.5 & 5.0 & 4.2 & 4.7 & 6.8 & 6.5 \\
\hline Poland & 3.0 & 3.1 & 3.6 & 4.3 & 4.2 & 3.5 & 3.5 \\
\hline Slovak Republic & 2.0 & 1.9 & 2.0 & 2.1 & 2.2 & 1.9 & 1.7 \\
\hline Switzerland & 4.2 & 4.4 & 4.6 & 3.7 & 2.3 & 5.1 & 4.4 \\
\hline UK & 3.3 & 4.2 & 4.0 & 4.5 & 4.2 & 3.6 & 3.7 \\
\hline Other & 12.4 & 11.5 & 12.9 & 13.9 & 12.3 & 11.4 & 10.3 \\
\hline Asia & 15.2 & 13.4 & 15.0 & 16.4 & 17.0 & 17.9 & 20.4 \\
\hline China & 4.7 & 4.4 & 4.8 & 5.1 & 5.6 & 6.4 & 6.2 \\
\hline Japan & 3.5 & 3.1 & 2.9 & 2.7 & 2.4 & 1.7 & 1.8 \\
\hline Other & 7.1 & 5.9 & 7.3 & 8.6 & 9.0 & 9.8 & 12.4 \\
\hline Western Hemisphere & 7.9 & 10.3 & 10.6 & 8.9 & 8.2 & 7.0 & 6.0 \\
\hline US & 5.3 & 7.2 & 6.5 & 4.5 & 4.2 & 3.8 & 3.2 \\
\hline Other & 2.6 & 3.1 & 4.1 & 4.4 & 4.0 & 3.3 & 2.9 \\
\hline Middle East and Africa & 2.6 & 3.9 & 3.8 & 3.3 & 4.0 & 4.4 & 3.9 \\
\hline Other & 0.2 & 0.4 & 0.3 & 0.1 & 0.1 & 0.1 & 0.1 \\
\hline
\end{tabular}

Source: State Customs Committee.

1/ Based on exports according to the Direction of Trade Statistics, which differ somewhat from those compiled by the Central Bank of Russia and shown in Table 23. 
Table 25. Russian Federation: Composition of Merchandise Exports, 1997-2003

\begin{tabular}{|c|c|c|c|c|c|c|c|}
\hline & 1997 & 1998 & 1999 & 2000 & 2001 & 2002 & 2003 \\
\hline & \multicolumn{7}{|c|}{ (In millions of U.S. dollars) } \\
\hline Total exports (f.o.b.) (including Belarus) & 85,036 & 71,313 & 72,885 & 103,093 & 99,970 & 106,712 & 133,450 \\
\hline Total exports (f.o.b.) 1/ (excluding Belarus) & 80,363 & 66,643 & 69,119 & 97,521 & 94,622 & 100,790 & 125,891 \\
\hline Food, beverage, and agricultural products & 1,407 & 1,187 & 764 & 1,299 & 1,460 & 2,177 & 2,689 \\
\hline Stone and ore & 784 & 821 & 579 & 712 & 550 & 583 & 724 \\
\hline Fuel products & 38,061 & 27,649 & 30,601 & 52,142 & 51,746 & 56,006 & 72,769 \\
\hline Oil and oil products & 20,819 & 13,619 & 18,828 & 34,361 & 32,775 & 38,585 & 50,748 \\
\hline Crude & 13,689 & 9,456 & 13,469 & 23,644 & 23,625 & 27,445 & 36,833 \\
\hline Oil products & 7,130 & 4,162 & 5,359 & 10,717 & 9,151 & 11,140 & 13,916 \\
\hline Gas & 15,644 & 12,696 & 10,850 & 16,118 & 17,243 & 15,359 & 19,312 \\
\hline Coal & 821 & 622 & 432 & 1,136 & 1,204 & 1,151 & 1,731 \\
\hline Other & 777 & 712 & 492 & 528 & 524 & 912 & 977 \\
\hline Chemicals (incld. pharmaceuticals and rubber) & 6,577 & 5,588 & 5,677 & 6,801 & 6,899 & 6,781 & 8,388 \\
\hline Leather & 383 & 372 & 187 & 237 & 175 & 187 & 232 \\
\hline Wood and paper products & 3,502 & 3,406 & 3,594 & 4,276 & 4,237 & 4,692 & 5,355 \\
\hline Textiles and clothing & 826 & 726 & 694 & 655 & 600 & 654 & 670 \\
\hline Gems and precious metals & 3,145 & 4,308 & 4,343 & 4,881 & 4,097 & 4,757 & 5,191 \\
\hline Metals & 16,715 & 14,708 & 14,147 & 16,682 & 13,929 & 14,347 & 17,387 \\
\hline Machines, equipment and instruments & 8,176 & 7,317 & 7,257 & 8,394 & 9,671 & 9,179 & 10,778 \\
\hline Other, including ceramics and glass & 786 & 562 & 1,275 & 1,442 & 1,260 & 1,427 & 1,709 \\
\hline Total exports (f.o.b.) 1/ (excluding Belarus) & \multicolumn{7}{|c|}{ (In percent of total exports) } \\
\hline Food, beverage, and agricultural products & 1.8 & 1.8 & 1.1 & 1.3 & 1.5 & 2.2 & 2.1 \\
\hline Stone and ore & 1.0 & 1.2 & 0.8 & 0.7 & 0.6 & 0.6 & 0.6 \\
\hline Fuel products & 47.4 & 41.5 & 44.3 & 53.4 & 54.7 & 55.6 & 57.8 \\
\hline Oil and oil products & 25.9 & 20.4 & 27.2 & 35.3 & 34.6 & 38.5 & 40.3 \\
\hline Crude & 17.0 & 14.2 & 19.5 & 24.3 & 25.0 & 27.4 & 29.3 \\
\hline Oil products & 8.9 & 6.2 & 7.8 & 11.0 & 9.7 & 11.1 & 11.1 \\
\hline Gas & 19.5 & 19.1 & 15.7 & 16.5 & 18.2 & 15.3 & 15.3 \\
\hline Coal & 1.0 & 0.9 & 0.6 & 1.2 & 1.3 & 1.1 & 1.4 \\
\hline Other & 1.0 & 1.1 & 0.7 & 0.5 & 0.6 & 0.6 & 0.8 \\
\hline Chemicals (incld. pharmaceuticals and rubber) & 8.2 & 8.4 & 8.2 & 7.0 & 7.3 & 6.8 & 6.7 \\
\hline Leather & 0.5 & 0.6 & 0.3 & 0.2 & 0.2 & 0.2 & 0.2 \\
\hline Wood and paper products & 4.4 & 5.1 & 5.2 & 4.4 & 4.5 & 4.7 & 4.3 \\
\hline Textiles and clothing & 1.0 & 1.1 & 1.0 & 0.7 & 0.6 & 0.7 & 0.5 \\
\hline Gems and precious metals & 3.9 & 6.5 & 6.3 & 5.0 & 4.3 & 4.7 & 4.1 \\
\hline Metals & 20.8 & 22.1 & 20.5 & 17.1 & 14.7 & 14.1 & 13.8 \\
\hline Machines, equipment and instruments & 10.2 & 11.0 & 10.5 & 8.6 & 10.2 & 9.1 & 8.6 \\
\hline Other, including ceramics and glass & 1.0 & 0.8 & 1.8 & 1.5 & 1.3 & 1.4 & 1.4 \\
\hline
\end{tabular}

Source: State Customs Committee.

1/ Excludes shuttle trade and other adjustments to the customs data that are included in Table 23. 
Table 26. Russian Federation: Origin of Imports, 1997-2003 1/

\begin{tabular}{|c|c|c|c|c|c|c|c|}
\hline & 1997 & 1998 & 1999 & 2000 & 2001 & 2002 & 2003 \\
\hline & \multicolumn{7}{|c|}{ (In millions of U.S. dollars) } \\
\hline Total imports & 53,039 & 43,581 & 30,278 & 33,879 & 41,883 & 46,174 & 57,263 \\
\hline CIS & 14,234 & 11,313 & 8,343 & 11,604 & 11,202 & 10,163 & 13,156 \\
\hline Belarus & 4,780 & 4,608 & 3,222 & 3,710 & 3,960 & 3,977 & 4,899 \\
\hline Kazakhstan & 2,743 & 1,884 & 1,391 & 2,197 & 2,018 & 1,946 & 2,475 \\
\hline Ukraine & 3,981 & 3,267 & 2,528 & 3,647 & 3,845 & 3,230 & 4,437 \\
\hline Other & 2,729 & 1,554 & 1,202 & 2,050 & 1,379 & 1,010 & 1,346 \\
\hline Non-CIS & 38,805 & 32,268 & 21,935 & 22,275 & 30,681 & 36,011 & 44,107 \\
\hline Europe & 26,074 & 20,268 & 13,779 & 14,087 & 19,128 & 22,779 & 27,768 \\
\hline Czech Republic & 586 & 530 & 343 & 366 & 467 & 564 & 712 \\
\hline Finland & 1,873 & 1,440 & 947 & 958 & 1,285 & 1,519 & 1,845 \\
\hline France & 1,592 & 1,595 & 1,233 & 1,188 & 1,538 & 1,896 & 2,342 \\
\hline Germany & 6,640 & 5,486 & 4,202 & 3,896 & 5,808 & 6,598 & 8,102 \\
\hline Hungary & 920 & 607 & 313 & 403 & 447 & 513 & 599 \\
\hline Ireland & 409 & 294 & 190 & 106 & 154 & 199 & 234 \\
\hline Italy & 2,640 & 1,819 & 1,157 & 1,211 & 1,715 & 2,228 & 2,402 \\
\hline Netherlands & 1,206 & 914 & 688 & 738 & 846 & 1,060 & 1,255 \\
\hline Poland & 1,354 & 1,046 & 602 & 715 & 962 & 1,300 & 1,713 \\
\hline Slovak Republic & 286 & 193 & 106 & 105 & 132 & 159 & 301 \\
\hline Switzerland & 524 & 426 & 309 & 271 & 391 & 417 & 535 \\
\hline UK & 1,481 & 1,221 & 676 & 860 & 1,003 & 1,120 & 1,439 \\
\hline Other & 6,563 & 4,697 & 3,013 & 3,270 & 4,380 & 5,205 & 6,289 \\
\hline Asia & 5,888 & 4,887 & 3,114 & 3,359 & 5,259 & 6,810 & 9,472 \\
\hline China & 1,261 & 1,160 & 894 & 948 & 1,646 & 2,401 & 3,295 \\
\hline Japan & 985 & 819 & 455 & 572 & 871 & 980 & 1,876 \\
\hline Other & 3,642 & 2,908 & 1,765 & 1,839 & 2,742 & 3,429 & 4,301 \\
\hline Western Hemisphere & 5,905 & 6,125 & 4,176 & 4,098 & 5,486 & 5,497 & 5,786 \\
\hline US & 4,061 & 4,114 & 2,387 & 2,694 & 3,253 & 2,980 & 2,959 \\
\hline Other & 1,844 & 2,011 & 1,789 & 1,404 & 2,233 & 2,517 & 2,827 \\
\hline Middle East and Africa & 709 & 641 & 620 & 584 & 627 & 760 & 909 \\
\hline \multirow[t]{2}{*}{ Other } & 229 & 347 & 246 & 147 & 181 & 164 & 172 \\
\hline & \multicolumn{7}{|c|}{ (In percent of total imports) } \\
\hline \multicolumn{8}{|l|}{ Total imports: } \\
\hline CIS & 26.8 & 26.0 & 27.6 & 34.3 & 26.7 & 22.2 & 23.0 \\
\hline Belarus & 9.0 & 10.6 & 10.6 & 11.1 & 9.5 & 8.8 & 8.6 \\
\hline Kazakhstan & 5.2 & 4.3 & 4.6 & 6.5 & 4.8 & 4.2 & 4.3 \\
\hline Ukraine & 7.5 & 7.5 & 8.3 & 10.8 & 9.2 & 7.0 & 7.7 \\
\hline Other & 5.1 & 3.6 & 4.0 & 6.1 & 3.3 & 2.2 & 2.4 \\
\hline Non-CIS & 73.2 & 74.0 & 72.4 & 65.7 & 73.3 & 77.8 & 77.0 \\
\hline Europe & 49.2 & 46.5 & 45.5 & 41.6 & 45.7 & 49.2 & 48.5 \\
\hline Czech Republic & 1.1 & 1.2 & 1.1 & 1.1 & 1.1 & 1.2 & 1.2 \\
\hline Finland & 3.5 & 3.3 & 3.1 & 2.8 & 3.1 & 3.3 & 3.2 \\
\hline France & 3.0 & 3.7 & 4.1 & 3.5 & 3.7 & 4.1 & 4.1 \\
\hline Germany & 12.5 & 12.6 & 13.9 & 11.5 & 13.9 & 14.3 & 14.1 \\
\hline Hungary & 1.7 & 1.4 & 1.0 & 1.2 & 1.1 & 1.1 & 1.0 \\
\hline Ireland & 0.8 & 0.7 & 0.6 & 0.3 & 0.4 & 0.4 & 0.4 \\
\hline Italy & 5.0 & 4.2 & 3.8 & 3.6 & 4.1 & 4.8 & 4.2 \\
\hline Netherlands & 2.3 & 2.1 & 2.3 & 2.2 & 2.0 & 2.3 & 2.2 \\
\hline Poland & 2.6 & 2.4 & 2.0 & 2.1 & 2.3 & 2.8 & 3.0 \\
\hline Slovak Republic & 0.5 & 0.4 & 0.4 & 0.3 & 0.3 & 0.3 & 0.5 \\
\hline Switzerland & 1.0 & 1.0 & 1.0 & 0.8 & 0.9 & 0.9 & 0.9 \\
\hline UK & 2.8 & 2.8 & 2.2 & 2.5 & 2.4 & 2.4 & 2.5 \\
\hline Other & 12.4 & 10.8 & 10.0 & 9.7 & 10.5 & 11.2 & 11.0 \\
\hline Asia & 11.1 & 11.2 & 10.3 & 9.9 & 12.6 & 14.7 & 16.5 \\
\hline China & 2.4 & 2.7 & 3.0 & 2.8 & 3.9 & 5.2 & 5.8 \\
\hline Japan & 1.9 & 1.9 & 1.5 & 1.7 & 2.1 & 2.1 & 3.3 \\
\hline Other & 6.9 & 6.7 & 5.8 & 5.4 & 6.5 & 7.4 & 7.5 \\
\hline Western Hemisphere & 11.1 & 14.1 & 13.8 & 12.1 & 13.1 & 11.9 & 10.1 \\
\hline US & 7.7 & 9.4 & 7.9 & 8.0 & 7.8 & 6.4 & 5.2 \\
\hline Other & 3.5 & 4.6 & 5.9 & 4.1 & 5.3 & 5.5 & 4.9 \\
\hline Middle East and Africa & 1.3 & 1.5 & 2.0 & 1.7 & 1.5 & 1.6 & 1.6 \\
\hline Other & 0.4 & 0.8 & 0.8 & 0.4 & 0.4 & 0.4 & 0.3 \\
\hline
\end{tabular}

Source: State Customs Committee.

1/ Based on imports according to the Direction of Trade Statistics, which differ somewhat from those compiled by the Central Bank of Russia and shown in Table 23. 
Table 27. Russian Federation: Composition of Merchandise Imports, 1997-2003 1/

\begin{tabular}{|c|c|c|c|c|c|c|c|}
\hline & 1997 & 1998 & 1999 & 2000 & 2001 & 2002 & 2003 \\
\hline & \multicolumn{7}{|c|}{ (In millions of U.S. dollars) } \\
\hline Total imports (c.i.f) (including Belarus) & 53,039 & 43,581 & 30,278 & 33,879 & 41,883 & 46,174 & 57,263 \\
\hline Total imports (c.i.f) 1/ (excluding Belarus) & 48,258 & 38,971 & 27,056 & 30,169 & 37,923 & 42,197 & 52,365 \\
\hline Food, beverage, tobacco and agricultural products & 12,715 & 10,266 & 7,674 & 6,977 & 8,736 & 9,832 & 11,304 \\
\hline Stone and ore & 764 & 590 & 426 & 671 & 660 & 668 & 799 \\
\hline Fuel products & 1,870 & 1,416 & 723 & 1,383 & 978 & 1,001 & 1,285 \\
\hline Chemicals (incld. pharmaceuticals and rubber) & 7,019 & 5,941 & 4,446 & 5,621 & 7,148 & 7,326 & 9,126 \\
\hline Leather & 155 & 96 & 58 & 89 & 190 & 172 & 186 \\
\hline Wood and paper products & 1,738 & 1,531 & 956 & 1,137 & 1,522 & 1,761 & 2,216 \\
\hline Textiles and clothing & 1,936 & 1,268 & 1,150 & 1,451 & 1,746 & 1,904 & 2,173 \\
\hline Gems and precious metals & 105 & 32 & 37 & 56 & 28 & 40 & 69 \\
\hline Metals & 3,311 & 2,665 & 1,955 & 2,492 & 2,760 & 2,597 & 3,620 \\
\hline Machines, equipment and instruments & 16,939 & 13,909 & 8,773 & 9,227 & 12,687 & 15,213 & 19,558 \\
\hline Other, including ceramics and glass & 1,708 & 1,259 & 857 & 1,067 & 1,467 & 1,683 & 2,030 \\
\hline Total imports (c.i.f) 1/ (excluding Belarus) & \multicolumn{7}{|c|}{ (In percent of total imports) } \\
\hline Food, beverage, tobacco and agricultural products & 26.3 & 26.3 & 28.4 & 23.1 & 23.0 & 23.3 & 21.6 \\
\hline Stone and ore & 1.6 & 1.5 & 1.6 & 2.2 & 1.7 & 1.6 & 1.5 \\
\hline Fuel products & 3.9 & 3.6 & 2.7 & 4.6 & 2.6 & 2.4 & 2.5 \\
\hline Chemicals (incld. pharmaceuticals and rubber) & 14.5 & 15.2 & 16.4 & 18.6 & 18.8 & 17.3 & 17.4 \\
\hline Leather & 0.3 & 0.2 & 0.2 & 0.3 & 0.5 & 0.4 & 0.4 \\
\hline Wood and paper products & 3.6 & 3.9 & 3.5 & 3.8 & 4.0 & 4.2 & 4.2 \\
\hline Textiles and clothing & 4.0 & 3.3 & 4.3 & 4.8 & 4.6 & 4.5 & 4.1 \\
\hline Gems and precious metals & 0.2 & 0.1 & 0.1 & 0.2 & 0.0 & 0.0 & 0.1 \\
\hline Metals & 6.9 & 6.8 & 7.2 & 8.3 & 7.3 & 6.2 & 6.9 \\
\hline Machines, equipment and instruments & 35.1 & 35.7 & 32.4 & 30.6 & 33.5 & 36.1 & 37.4 \\
\hline Other, including ceramics and glass & 3.5 & 3.2 & 3.2 & 3.5 & 3.9 & 4.0 & 3.9 \\
\hline
\end{tabular}

Source: State Customs Committee.

1/ Excludes shuttle trade and other adjustments to the customs data that are included in Table 23. 
Table 28. Russian Federation: Foreign Currency Disbursements to the Federal Government, 1999-2003 (In millions of U.S. dollars)

\begin{tabular}{|c|c|c|c|c|c|}
\hline Creditors & 1999 & 2000 & 2001 & 2002 & 2003 \\
\hline Multilateral & 1,209 & 584 & 428 & 426 & 352 \\
\hline IMF & 641 & 0 & 0 & 0 & 0 \\
\hline World Bank & 545 & 552 & 377 & 410 & 233 \\
\hline EBRD & 22 & 32 & 51 & 16 & 119 \\
\hline Other & 0 & 0 & 0 & 0 & 0 \\
\hline Bilateral and suppliers/other commercial & 1,490 & 454 & 198 & 430 & 442 \\
\hline Tied & 1,058 & 254 & 98 & 380 & 442 \\
\hline Untied & 432 & 200 & 100 & 50 & 0 \\
\hline Bonds & 0 & 0 & 0 & 0 & 0 \\
\hline Resident purchases & 0 & 0 & 0 & 0 & 0 \\
\hline Nonresident purchases & 0 & 0 & 0 & 0 & 0 \\
\hline Total & 2,699 & 1,038 & 626 & 856 & 794 \\
\hline (Excluding IMF) & 2,057 & 1,038 & 626 & 856 & 794 \\
\hline \multicolumn{6}{|l|}{ Memorandum items: } \\
\hline Minfin bonds & 0 & 0 & 0 & 0 & 0 \\
\hline OGNZ & 0 & 0 & 0 & 0 & 0 \\
\hline
\end{tabular}

Source: Russian authorities. 
Table 29. Russian Federation: Nonsovereign/Sector Financial Account, 1997-2003 1/ (In millions of U.S. dollars)

\begin{tabular}{|c|c|c|c|c|c|c|c|}
\hline & 1997 & 1998 & 1999 & 2000 & 2001 & 2002 & 2003 \\
\hline Direct investment & 1,681 & 1,492 & 1,102 & -463 & 216 & -72 & $-3,002$ \\
\hline Abroad & $-3,184$ & $-1,270$ & $-2,208$ & $-3,177$ & $-2,533$ & $-3,533$ & $-9,727$ \\
\hline In Russia & 4,865 & 2,761 & 3,309 & 2,714 & 2,748 & 3,461 & 6,725 \\
\hline Portfolio investment & 2,198 & 878 & -253 & 0 & 1,134 & 2,793 & $-3,263$ \\
\hline Assets & -156 & -257 & 254 & -411 & 77 & -796 & $-2,543$ \\
\hline Equity & 32 & -10 & 5 & -40 & -60 & 85 & -13 \\
\hline Debt securities & -189 & -247 & 249 & -371 & 137 & -880 & $-2,529$ \\
\hline Liabilities & 2,354 & 1,136 & -507 & 411 & 1,057 & 3,589 & -720 \\
\hline Equity & 1,265 & 714 & -287 & 150 & 542 & 2,626 & 413 \\
\hline Banks & 93 & 33 & -10 & 2 & 14 & 588 & 56 \\
\hline Nonfinancial enterprises & 1172 & 681 & -277 & 148 & 529 & 2,038 & 357 \\
\hline Debt securities & 1089 & 422 & -220 & 261 & 515 & 962 & -1133 \\
\hline Local governments & 872 & 533 & -175 & -427 & -447 & -63 & 129 \\
\hline Issue & 867 & 499 & 0 & 0 & 0 & 0 & 0 \\
\hline Redemption & -36 & -82 & -118 & -258 & -326 & -93 & -77 \\
\hline Secondary market & -2 & 0 & -190 & -273 & -159 & 11 & 189 \\
\hline Interest reinvestment & 43 & 116 & 133 & 104 & 38 & 20 & 16 \\
\hline Monetary authorities & $\ldots$ & $\ldots$ & $\ldots$ & $\ldots$ & -36 & 0 & 0 \\
\hline Banks & 110 & -262 & -97 & 527 & 780 & 239 & -480 \\
\hline Nonfinancial enterprises & 107 & 150 & 52 & 160 & 217 & 786 & -782 \\
\hline Other investments & $-12,010$ & $-13,129$ & $-13,131$ & $-16,133$ & $-5,343$ & $-5,002$ & 12,435 \\
\hline Assets & $-27,653$ & $-12,386$ & $-11,341$ & $-15,409$ & $-9,654$ & $-14,593$ & $-15,320$ \\
\hline Cash foreign currency and deposits & $-13,112$ & 1,804 & $-2,763$ & $-4,695$ & $-1,059$ & 1,187 & 7,519 \\
\hline Trade credit (net) & -696 & $-5,388$ & $-3,322$ & $-4,245$ & 475 & $-1,697$ & $-4,012$ \\
\hline Loans & $-2,639$ & -334 & 279 & -440 & $-1,041$ & $-1,562$ & $-2,302$ \\
\hline Banks & $-2,164$ & 40 & 409 & -395 & -593 & $-1,493$ & -684 \\
\hline Nonfinancial enterprises & -475 & -373 & -129 & -45 & -368 & -3 & $-1,618$ \\
\hline Monetary authorities & $\ldots$ & $\ldots$ & $\ldots$ & $\ldots$ & -80 & -66 & 0 \\
\hline Arrears & 22 & -148 & -47 & 188 & -118 & -10 & -40 \\
\hline Banks & 22 & -148 & -40 & 188 & -118 & -10 & -40 \\
\hline Nonfinancial enterprises & 0 & 0 & -6 & 0 & 0 & 0 & 0 \\
\hline \multicolumn{8}{|l|}{$\begin{array}{l}\text { Changes in the stock of nonrepatriated export proceeds } \\
\text { and nonrepatriated import advances, remittances }\end{array}$} \\
\hline against fictitious transactions in securities & $-11,591$ & $-7,959$ & $-5,051$ & $-5,293$ & $-6,388$ & $-12,244$ & $-15,435$ \\
\hline Other & 363 & -361 & -438 & -923 & $-1,523$ & -268 & $-1,051$ \\
\hline Liabilities & 15,643 & -743 & $-1,790$ & -724 & 4,311 & 9,591 & 27,756 \\
\hline Cash foreign currency and deposits & 4,278 & $-2,975$ & 111 & 727 & 1,530 & 512 & 3,855 \\
\hline Trade credit & 0 & 0 & 0 & 0 & 0 & 0 & 0 \\
\hline Loans & 10,833 & 1,983 & $-1,958$ & $-1,599$ & 3,444 & 9,343 & 24,046 \\
\hline Local governments & 358 & 392 & 7 & -267 & 280 & -4 & -112 \\
\hline Banks & 3,840 & $-3,407$ & $-1,517$ & -397 & 411 & 2,300 & 7,513 \\
\hline Nonbank financial organizations & $-1,516$ & 0 & 0 & 0 & 0 & 0 & 0 \\
\hline Nonfinancial enterprises & 8,152 & 4,997 & -448 & -935 & 769 & 8,334 & 15,128 \\
\hline Monetary authorities (excluding IMF credits) & & & & & 1,984 & $-1,288$ & 1,517 \\
\hline Arrears & 3 & 927 & 258 & -48 & 0 & -366 & -11 \\
\hline Local governments & 0 & 228 & -79 & -90 & -5 & -3 & -7 \\
\hline Banks & 3 & 698 & 337 & 42 & 5 & -363 & -4 \\
\hline Nonfinancial enterprises & 0 & 0 & 0 & 0 & 0 & 0 & 0 \\
\hline Other & 529 & -677 & -200 & 196 & -663 & 103 & -135 \\
\hline Total (net) & $-8,131$ & $-10,759$ & $-12,282$ & $-16,596$ & $-3,993$ & $-2,281$ & 6,170 \\
\hline
\end{tabular}

Source: Central Bank of Russia, staff calculations.

1/ Data are on an accrual basis and therefore differ from those shown in Table 23. 
Table 30. Russian Federation: External Debt, 1997-2003 1/ (In billions of U.S. dollars)

\begin{tabular}{|c|c|c|c|c|c|c|c|}
\hline & 1997 & 1998 & 1999 & 2000 & 2001 & 2002 & 2003 \\
\hline \multicolumn{8}{|l|}{ Sovereign debt } \\
\hline A. Russian-era foreign currency debt (post 1/1/1992) & 35.6 & 56.0 & 51.7 & 46.4 & 39.2 & 37.9 & 33.5 \\
\hline $\begin{array}{l}\text { Medium and long term } \\
\text { Multilateral Creditors } \\
\text { IMF (including loans to the Central Bank of Russia) } \\
\text { World Bank } \\
\text { Other } \\
\text { Official creditors } \\
\text { Eurobonds } \\
\text { OGNZ } \\
\text { Minfin bonds (Minfins VI, VII and VIII) } \\
\text { Commercial creditors (includes financial institutions) } \\
\text { Short term 1/ }\end{array}$ & $\begin{array}{r}18.7 \\
13.2 \\
5.3 \\
0.2 \\
7.6 \\
4.5 \\
0.0 \\
3.5 \\
1.3\end{array}$ & $\begin{array}{r}26.0 \\
19.4 \\
6.4 \\
0.2 \\
9.7 \\
16.0 \\
0.6 \\
3.5 \\
0.2\end{array}$ & $\begin{array}{r}22.3 \\
15.3 \\
6.8 \\
0.2 \\
9.8 \\
15.6 \\
0.4 \\
3.5 \\
0.1\end{array}$ & $\begin{array}{r}18.9 \\
11.6 \\
7.1 \\
0.2 \\
8.5 \\
15.5 \\
0.0 \\
3.5 \\
0.0\end{array}$ & $\begin{array}{r}14.9 \\
7.6 \\
7.1 \\
0.2 \\
6.4 \\
14.4 \\
0.0 \\
3.5 \\
0.0\end{array}$ & $\begin{array}{r}14.0 \\
6.6 \\
7.1 \\
0.3 \\
5.7 \\
14.7 \\
0.0 \\
3.5 \\
0.0\end{array}$ & $\begin{array}{r}11.7 \\
5.1 \\
6.3 \\
0.3 \\
4.9 \\
13.4 \\
0.0 \\
3.5 \\
0.0\end{array}$ \\
\hline $\begin{array}{l}\text { B. Soviet-era foreign currency debt (pre 1/1/1992) } \\
\text { Multilateral Creditors } \\
\text { Official creditors } \\
\text { Paris Club } \\
\text { COMECON (including clearing accounts) } \\
\text { Other, including non-Paris Club bilateral } \\
\text { of which: arrears } \\
\text { of which: principal arrears } \\
\text { interest arrears }\end{array}$ & $\begin{array}{r}99.0 \\
0.0 \\
56.9 \\
37.6 \\
14.9 \\
4.4 \\
\ldots\end{array}$ & $\begin{array}{r}102.8 \\
0.0 \\
59.4 \\
40.0 \\
14.8 \\
4.6 \\
4.0\end{array}$ & $\begin{array}{r}103.6 \\
0.0 \\
58.3 \\
38.7 \\
14.9 \\
4.8 \\
4.5\end{array}$ & $\begin{array}{r}93.3 \\
0.0 \\
58.4 \\
39.0 \\
14.0 \\
5.4 \\
5.3\end{array}$ & $\begin{array}{r}88.2 \\
0.0 \\
53.3 \\
36.3 \\
11.3 \\
5.7 \\
5.6\end{array}$ & $\begin{array}{r}81.5 \\
0.0 \\
48.9 \\
39.2 \\
4.1 \\
5.6 \\
5.6\end{array}$ & $\begin{array}{r}82.2 \\
0.0 \\
51.5 \\
42.9 \\
3.6 \\
5.0 \\
5.0\end{array}$ \\
\hline Commercial creditors & 34.4 & 35.8 & 37.7 & 6.7 & 6.8 & 4.5 & 4.6 \\
\hline Financial institutions & 29.7 & 31.2 & 32.8 & 1.8 & 1.9 & 1.9 & 2.0 \\
\hline of which: arrears & $\ldots$ & 2.1 & 3.8 & 1.8 & 1.9 & 1.9 & 2.0 \\
\hline Other & 4.2 & 4.1 & 4.1 & 4.1 & 4.1 & 2.4 & 2.4 \\
\hline of which: arrears & $\ldots$ & 4.1 & 4.1 & 4.1 & 4.1 & 2.1 & 2.4 \\
\hline Credits contracted by entities other than VEB & 0.5 & 0.5 & 0.8 & 0.8 & 0.8 & 0.2 & 0.2 \\
\hline Eurobonds & 0.1 & 0.0 & 0.0 & 20.9 & 20.9 & 22.3 & 22.3 \\
\hline Minfin bonds (Minfins III, IV and V) & 7.6 & 7.6 & 7.6 & 7.3 & 7.3 & 5.8 & 3.8 \\
\hline of which: arrears & 0.0 & 0.0 & 1.3 & 0.1 & 0.0 & 0.0 & 0.0 \\
\hline C. Total sovereign foreign currency debt $(=\mathrm{A}+\mathrm{B})$ & 134.6 & 158.7 & 155.4 & 139.7 & 127.4 & 119.4 & 115.7 \\
\hline Medium and long term & 127.8 & 151.1 & 146.1 & 132.0 & 119.4 & n.a. & n.a. \\
\hline Short term & 6.8 & 7.6 & 9.3 & 7.6 & 8.1 & n.a. & n.a. \\
\hline \multicolumn{8}{|l|}{ Memo items: } \\
\hline $\begin{array}{l}\text { Ministry of Finance obligations owed } \\
\text { indirectly to the Central Bank }\end{array}$ & 0.0 & 2.3 & 6.4 & 6.4 & 6.4 & 6.2 & 6.2 \\
\hline
\end{tabular}

Source: Russian authorities.

1/ Government securities are reported at face value. Some totals may not add up due to rounding. 